\title{
Trends and correlates of meeting 24-hour movement guidelines: a 15-year study among 167,577 Thai adults
}

Nucharapon Liangruenrom ${ }^{1,2}$, Dorothea Dumuid ${ }^{3}$, Melinda Craike ${ }^{1,4}$, Stuart J. H. Biddle ${ }^{5}$ and Zeljko Pedisic ${ }^{1 *}$

\begin{abstract}
Background: Time spent in physical activity (PA), sedentary behaviour (SB), and sleep always takes up the whole day. New public health guidelines combining recommendations for PA, SB, and sleep have been issued in several countries. Thailand was the first country to release the 24-h guidelines for adults. Currently, there is no evidence on the population prevalence of meeting 24-h movement guidelines in Thailand. This study, therefore, aimed to determine 15-year trends and associations of meeting 24-h movement guidelines among Thai adults.

Method: We analysed cross-sectional data from 2001, 2004, 2009, and 2015 Thai Time-Use Surveys, coded using the International Classification of Activities for Time-Use Statistics (ICATUS). All ICATUS-based activities were categorised into moderate-to-vigorous PA (MVPA), light PA (LPA), SB, and sleep based on a previously developed classification system. A total of 167,577 adult participants were included. The participants were classified according to the Thai 24-h movement guidelines into meeting or not meeting the following criteria: 1) $\geq 150 \mathrm{~min} /$ week of MVPA; 2) interrupting SB every $2 \mathrm{~h} ; 3$ ) sleeping $7-9 \mathrm{~h}$ per day; and 4) adhering to all three guidelines.
\end{abstract}

Results: In 2015, the prevalence of adults who met the MVPA, SB, sleep, and overall recommendations was 81.7, $44.6,56.4$, and $21.3 \%$, respectively. A significant linear increase was found for the prevalence of meeting the SB recommendation, while the prevalence meeting the MVPA, sleep, and overall recommendations was lowest in 2001, peaked in 2004 or 2009, and declined in 2015. The lowest odds for meeting the 24-h guidelines were found among males, those living in urban areas, inhabitants of Bangkok and South Thailand, unemployed, and those with low education level.

Conclusions: Despite promising trends in the prevalence of meeting PA, SB, and sleep recommendations, a majority of Thai adults still do not meet the overall 24-h movement guidelines. Further actions are needed to promote more MVPA, less SB, and adequate sleep in Thai adults, particularly among males, those living in urban areas, inhabitants of Bangkok and South Thailand, unemployed, and those with low education level.

Keywords: Time-use data, Physical activity, Sedentary behaviour, Sleep, ICATUS, Time-use epidemiology

\section{Background}

Given that everyone has a fixed 24-h budget in a day and the time spent in physical activity (PA), sedentary

\footnotetext{
* Correspondence: zeljko.pedisic@vu.edu.au

${ }^{1}$ Institute for Health and Sport, Victoria University, PO Box 14428, Melbourne, VIC 8001, Australia

Full list of author information is available at the end of the article
}

behaviour $(\mathrm{SB})$, and sleep always takes up the whole day, any change in the time spent in one of these behaviours necessarily affects the remaining behaviours [1-4]. Recent epidemiological research has therefore often considered these behaviours collectively in relation to health. Growing evidence shows that high PA, low sedentary time, and adequate sleep duration are collectively 
associated with a range of health benefits, such as lower body mass index (BMI), low waist circumference, and high aerobic fitness [5-10]. Based on the emerging evidence and a better understanding of the importance of considering these behaviours holistically, new public health guidelines that combine recommendations for PA, SB, and sleep have been issued in several countries [11-19]. Canada pioneered the development of such guidelines, and in 2016 they launched the first national 24-h movement guidelines [16]. Soon after, following the Canadian example, Australia, Finland, New Zealand, South Africa, and Thailand issued their 24-h movement guidelines [11-15]. To date, a few additional countries, such as Croatia and the United Kingdom, have made initial steps in the process of adopting similar public health guidelines [17, 18]. The World Health Organization (WHO) supported the integrative approach to movement behaviours and has recently released 24-h guidelines for children under 5 years of age [19].

Time-use surveys provide comprehensive data on common activities that the general population performs in a day [20]. Since the 1960s, time-use data have been collected worldwide in over 85 countries [20]. With contextualised information across life domains and international availability, these surveys have been a valuable source of data for epidemiological research on movement behaviours [21]. They are considered to provide sufficiently valid and reliable PA, SB, and sleep estimates for observational studies [3, 21-26].

To date, time-use data have been used to examine population prevalence and correlates of $\mathrm{PA}$ and $\mathrm{SB}$, particularly in high-income countries [23, 27-37]. Some studies also used time-use survey data to explore the prevalence of $\mathrm{PA} / \mathrm{SB}$ or meeting $\mathrm{PA}$ recommendations and trends over time [38-41]. Assessing the prevalence of meeting the 24-h movement guidelines has started to gain momentum in national health monitoring studies [42], and several studies have examined associations of meeting the guidelines with health and psychological wellbeing among youth [43-45]. The 24-h movement guidelines are a new benchmark for assessing healthy use of time in the population. Determining the prevalence of meeting these new public health guidelines is essential to inform future health promotion and disease prevention policies and interventions. However, only a few previous studies have assessed the prevalence and correlates of meeting the 24-h movement guidelines in national-representative samples of adults [42, 43, 45]. Further, no studies have employed data from time-use surveys for this purpose.

Thailand is an upper-middle income country, and its Government has a progressive approach for improving population health. For example, universal health coverage was introduced in 2002, and significant progress was made in the primary prevention of chronic diseases, particularly by taking actions to promote PA in the population [46, 47]. Thailand recently released the 1st National Strategic Plan for the Promotion of Physical Activity (2018-2030), and its Ministry of Public Health took part in issuing the Bangkok Declaration on Physical Activity for Global Health and Sustainable Development [48, 49]. The Thai National Strategic Plan for the Promotion of Physical Activity was primarily based on the Global Recommendations on Physical Activity for Health, developed by the WHO [13, 50]. However, taking into account emerging evidence on collective health impacts of PA, SB, and sleep, Thailand issued holistic, national 24-h movement guidelines for five different population groups in 2017 [13]. These target population groups include: 1 ) pregnant and postpartum women; 2 ) early years ( $0-5$ years); 3$)$ school-aged children and adolescents (617 years); 4) adults (18-59 years); and 5) older adults (60 years and older). To our knowledge, Thailand was the first country to issue 24-h movement guidelines, including PA, SB, and sleep recommendations, for adults, older adults, and pregnant and postpartum women. This is consistent with the progressive thinking and strong commitment of the Thai Government to improve national preventative healthcare.

Currently, there is no evidence on the population prevalence of meeting 24-h movement guidelines in Thailand. There is also a lack of evidence on PA and SB trends in the Thai population [51]. In addition, the authors are not aware of any studies that have examined the temporal changes of factors associated with meeting the integrated movement guidelines. This study, therefore, aimed to determine 15-year trends of meeting 24-h movement guidelines and to examine how associations between sociodemographic variables and meeting these recommendations have changed over time.

Previous research from other countries has shown that, in the last few decades, in some countries, there has been a clear shift from physically active to sedentary lifestyles [39], while sleep duration in adult populations has generally remained constant [52-54]. We, therefore, hypothesised that among Thai adults PA will have decreased, SB increased, and sleep duration remained stable since 2001.

\section{Methods}

\section{Thai national time-use survey}

The National Statistical Office (NSO) conducted crosssectional Thai National Time-Use Surveys in 2001, 2004, 2009, and 2015 [55-58]. The NSO randomly selected private households by using the three-stage stratified sampling method [55-58]. The stratification was according to five Thai regions (Bangkok, Central, North, North-East, and South), 77 provinces, and municipal 
and non-municipal areas called Enumerations (EAs) [55-58]. The Municipality Act, B.E. 2496 (1953) divides household areas into municipal or urban areas, where a single administrative district has powers of selfgovernment (also known as cities), and non-municipal or rural areas, such as villages [59]. A random sample of EAs was selected within each province. A random sample of households was selected from a list of dwellings within each selected EA. One member from each of the selected households was randomly selected. Among the selected household members, data were collected using a combination of a direct interview and self-administered questionnaires. The participants were asked to report all activities they engaged in on a selected day, including the start and end time for each activity. The NSO trained officers recorded and coded participants' daily activities over a 24-h day (from 0:00 to 24:00 h) using the ICATUS coding rules and a computer-assisted coding device $[60,61]$. The reported activities were coded for 10-min intervals, except in the survey year 2001, when the reported activities were recorded and coded with no fixed time intervals. The surveys captured main (primary) activities, and if more than one activity was performed simultaneously a secondary activity was recorded. The surveys also included household and individual questionnaires to gather sociodemographic information about respondents. This study analysed time-use data from all four survey years.

\section{Ethics approval and consent}

The NSO is a state agency responsible for a production of statistical data for national development. The agency is enacted by the Official Information Act, B.E. 2540 (1997) [59]. In accordance with the Official Information Act, participants provided informed consent prior to the survey. and their anonymity and confidentiality of their data were protected by the legislation [59]. For this study, we obtained a permission from the NSO to access microdata of four time-use surveys for research purposes only.

\section{Participants}

This study included only adult participants (18-59 years old) with complete 24-h data. We applied this age range based on the Thai Labour Protection Act (No. 6), B.E. 2560 (2017), which specifies the retirement age for adults at 60 years and above [62]. Retirement is a major event in life that very often significantly affects time-use patterns, including PA, SB, and sleep, as well as many other components of time use [63]. The compulsory retirement age is, therefore, commonly considered as the cut-off point between adulthood and older adulthood. Additionally, Thai 24-h movement recommendations are different for adults and older adults, and the age threshold for older adults is 60 years [13]. The total pooled sample size from four survey years was 167,577 $(n=37,702$ [50.2\% females] in 2001; $n=37,544$ [49.9\% females] in 2004; $n=45,751$ [50.8\% females] in 2009; and $n=46,580$ [51.0\% females] in 2015).

\section{Measures and data processing}

The self-reported time-use data were coded using the ICATUS classification. The time-use codes from the survey year 2001 were based on the Draft ICATUS comprising 10 major groups of activities [57]. The other survey years used the Trial ICATUS consisting of 15 major groups of activities $[55,56,58]$. The Trial ICATUS is a revised version of the Draft ICATUS [61]. One author (NL) harmonised activities in the Draft ICATUS with corresponding activities from the Trial ICATUS. Once all activities were matched, they were categorised into sleep, SB, LPA, and MVPA based on the classification system previously developed for the Trial ICATUS [64]. A detailed description of the method used to classify ICATUS activities can be found elsewhere [64]. Due to insufficient information provided for occupational and travel-related activities in ICATUS, this classification system did not apply to such activities. Instead, the reported occupational and travel-related activities were linked with additional information on occupations and location or mode of travel of respondents available in the household questionnaires, according to the procedures suggested in previous studies [41, 64, 65]. The occupations of respondents were linked with a summary MET previously assigned to the occupation list of 2008 International Standard Classification of Occupations (ISCO-08) [66]. For travel-related codes, the location or mode of travel of respondents was linked with a summary MET assigned to travelling modes available in a previous study [65].

Once all ICATUS-based activities from 2001, 2004, 2009, and 2015 were categorised into sleep, SB, LPA, and MVPA, the time (in minutes) spent in each of the behaviours was calculated. The time-use surveys only captured 1 day of data for each participant, which was assumed to represent their average daily behaviour. Each participant was categorised as either "meeting" or "not meeting" the Thai 24-h movement guidelines for adults. The guidelines include the following recommendations: 1) to engage in at least $150 \mathrm{~min} /$ week of moderate PA, or $75 \mathrm{~min} /$ week of vigorous $\mathrm{PA}$, or an equivalent combination of the two PA intensities; 2) to interrupt SB every $2 \mathrm{~h}$; and 3) to sleep between 7 and $9 \mathrm{~h}$ per day [13]. The guidelines also include an MVPA recommendation for additional health benefits, defined as at least $300 \mathrm{~min} /$ week of moderate PA, or $150 \mathrm{~min} /$ week of vigorous PA, or an equivalent combination of the two PA intensities [13]. The participants who met all three 
recommendations for MVPA, SB, and sleep, were categorised as meeting the overall 24-h movement guidelines.

Given that several ICATUS activity groups are broad, it is not feasible to distinguish activities of moderate and vigorous intensity [64]. Following the previously developed classification of ICATUS activities [64], a combination of moderate and vigorous intensities (i.e., MVPA) was used. The amount of time spent in MVPA, SB, and sleep (including napping) was calculated and used to determine whether participants met the recommendations.

For meeting the MVPA recommendation, participants who engaged in a minimum of $150 \mathrm{~min} /$ week of MVPA, which translates into an average of approximately 22 $\mathrm{min} /$ day, were categorised as meeting the MVPA recommendation. For the SB recommendation, participants who spent no more than $120 \mathrm{~min}$ in a single SB activity or consecutive SB activities were classified as meeting the recommendation. From the available data, it was clear that these participants did not engage in SB for more than 120 consecutive minutes, because their SB was interrupted by either PA or sleep. This, however, provides a conservative estimate of the prevalence of meeting the SB guideline, because no data was available on possible interruptions of SB within the 10-min reference periods. Finally, participants who slept between 420 and $540 \mathrm{~min}$ per day were classified as meeting the sleep recommendation.

Recommendation for muscle-strengthening activities is also a part of the guidelines [13]. However, in ICATUSbased time-use surveys, these activities are broadly grouped in fitness-related category which includes yoga and other aerobic activities [60]. It was, therefore, not possible to determine whether participants met the recommendation for muscle-strengthening activities.

\section{Study correlates}

Sociodemographic variables were selected a priori based on previous evidence of their associations with PA, SB, and sleep in adults [54,67-74] and availability of data from the Thai National Time-Use Surveys. We extracted self-reported data on eight sociodemographic variables that were available in all four survey years. These included sex (male, female), age groups (18-29 years, 3039 years, $40-49$ years, and 50-59 years), region (Bangkok, Central, North, North-east, and South), household area (municipal or urban area, non-municipal or rural area), employment status (employed, unemployed), highest education level (none, primary school, secondary school, higher education, and unspecified), marital status (never married, married, and formerly married), and religion (Buddhist, nonBuddhist). Our decision to examine religion as a potential correlate was based on the Kim and Sobal [73] study that found a significant association between religious denomination and PA, and on the mapping review by Langøien et al. [74] that suggested more studies are needed to investigate how different religious affiliations are associated with PA and SB.

\section{Data analysis}

All data were weighted using the population weights provided by NSO, to represent the Thai adult population. Additional weights were used to adjust for slight discrepancies from the assumed uniform distribution of surveys across the 7 days of the week. Percentages (and their 95\% confidence intervals [CIs]) of participants meeting MVPA, SB, Sleep, and overall 24-h movement recommendations were computed for the overall sample and by the sociodemographic categories. A series of multivariate logistic regressions was performed to examine the associations between the sociodemographic variables and participants' compliance within the 24-h movement guidelines. The odds ratios (ORs) were adjusted for sex, age groups, household area, region, marital status, religion, employment status, and education level, and their 95\% CIs were calculated. A series of mixed-effects meta-regression analyses with Restricted Maximum Likelihood Estimation (REML) were used to establish the time-trends in prevalence estimates and the time-trends in the associations of sociodemographic variables with meeting the 24-h movement guidelines. Linear and quadratic functions were fitted to each timetrend, and the model with the lower Akaike Information Criterion (AIC) value was selected. The statistical significance level was set at $p<0.05$. All analyses were conducted in R ( $\mathrm{R}$ Foundation for Statistical Computing, Vienna, Austria), using "questionr" and "metafor" packages $[75,76]$.

\section{Results}

\section{Sample characteristics}

The weighted sample included nearly equal males and females (Table 1). In 2001, the most represented age group were young adults (18-29 years old), but the prevalence of older age groups continuously increased throughout the 15-year period. In the first three survey rounds, around two thirds of participants lived in rural areas. From 2009 to 2015 there was a large reallocation from rural to urban areas, resulting in a nearly equal split in the final survey year. In 2001, the most represented region in the sample was North-East (33.8\%), while the largest percentage of participants in 2015 were from the Central region (30.4\%). In all survey years, most participants were married, Buddhist, and employed. In 2001, primary school was the highest level of education for most participants (60.2\%). However, the level of education attainment increased during the study 
Table 1 Characteristics of the weighted samples in the 2001, 2004, 2009, and 2015 Thai time-use surveys

\begin{tabular}{|c|c|c|c|c|}
\hline Sociodemographic variable & 2001 & 2004 & 2009 & 2015 \\
\hline Total $(n)$ & 37,702 & 37,544 & 45,751 & 46,580 \\
\hline \multicolumn{5}{|l|}{ Sex $(\%)$} \\
\hline Male & 49.8 & 50.1 & 49.2 & 49.0 \\
\hline Female & 50.2 & 49.9 & 50.8 & 51.0 \\
\hline \multicolumn{5}{|l|}{ Age groups (\%) } \\
\hline $18-29$ & 35.4 & 34.3 & 29.6 & 27.5 \\
\hline $30-39$ & 27.4 & 27.2 & 26.2 & 24.5 \\
\hline $40-49$ & 22.5 & 22.8 & 25.5 & 26.1 \\
\hline $50-59$ & 14.7 & 15.7 & 18.7 & 22.0 \\
\hline \multicolumn{5}{|l|}{ Household area (\%) } \\
\hline Urban & 32.3 & 34.6 & 32.7 & 46.9 \\
\hline Rural & 67.7 & 65.4 & 67.3 & 53.1 \\
\hline \multicolumn{5}{|l|}{ Region (\%) } \\
\hline Bangkok & 12.1 & 14.2 & 11.1 & 15.0 \\
\hline Central & 22.9 & 23.7 & 24.5 & 30.4 \\
\hline North & 19.0 & 18.2 & 18.3 & 16.3 \\
\hline North-East & 33.8 & 31.6 & 32.8 & 25.2 \\
\hline South & 12.3 & 12.2 & 13.2 & 13.2 \\
\hline \multicolumn{5}{|l|}{ Marital status (\%) } \\
\hline Never married & 24.4 & 24.6 & 23.1 & 27.7 \\
\hline Married & 69.7 & 69.6 & 70.1 & 64.5 \\
\hline Formerly married & 5.9 & 5.7 & 6.8 & 7.8 \\
\hline \multicolumn{5}{|l|}{ Religion (\%) } \\
\hline Buddhist & 95.1 & 95.3 & 95.1 & 95.4 \\
\hline Non-Buddhist & 4.9 & 4.7 & 4.9 & 4.6 \\
\hline \multicolumn{5}{|l|}{ Employment status (\%) } \\
\hline Employed & 82.4 & 82.4 & 83.1 & 80.8 \\
\hline Unemployed & 17.6 & 17.6 & 16.9 & 19.2 \\
\hline \multicolumn{5}{|l|}{ Highest education level (\%) } \\
\hline None & 3.2 & 3.1 & 4.5 & 9.8 \\
\hline Primary & 60.2 & 54.6 & 47.9 & 35.2 \\
\hline Secondary & 25.7 & 29.2 & 30.0 & 31.5 \\
\hline Higher education & 10.7 & 12.7 & 17.3 & 23.0 \\
\hline Unspecified & 0.2 & 0.3 & 0.2 & 0.5 \\
\hline
\end{tabular}

period, as more people completed secondary or higher education.

\section{Prevalence of meeting 24-h movement guidelines}

In 2015, the prevalence of Thai adults who met 24-h movement guidelines was $21.3 \%$ (95\% CI: 20.9, 21.7). For the whole sample and for most sociodemographic groups, the sample prevalence rose from 2001 to its peak in 2009, and then declined in 2015. However, this inverted U-shaped trend was found to be significant only among females (Fig. 1), those who were 30-39 years of age, residents of rural areas, inhabitants of the Central and North-East region, those who have never been married or who were formerly married, unemployed, and those who had secondary or higher education. The prevalence of meeting 24-h guidelines showed significant incremental increase from 2001 to 2015 ( $p$-value for linear trend < 0.05) among males (Fig. 2), those aged $40-49$ years, and those who lived in Bangkok or the South region.

\section{Associations of sociodemographic characteristics with meeting the overall $24-\mathrm{h}$ movement guidelines}

Females had 53, 60, 73, and 43\% higher odds of meeting the overall guidelines than males in 2001, 2004, 2009, and 2015, respectively (Table 2). Thai adults who lived in Bangkok had the lowest odds of meeting the overall guidelines, while those from the North region had the highest odds in all survey years except in 2009. In most survey years, significantly higher odds of meeting the overall guidelines were also found for those who were married or formerly married (compared to those who have never been married) and employed (compared to unemployed). An inverted U-shaped trend was found for the odds of meeting the guidelines for the higher education group, where the odds increased from 2001 to 2009 and then decreased in $2015(p=0.046)$.

\section{Prevalence of meeting the MVPA recommendations}

In 2015, the prevalence of Thai adults meeting the MVPA recommendations was $81.7 \%$ (95\% CI: 81.3, 82.1). For the whole sample, and for all but one sociodemographic group, the sample prevalence of adults meeting the MVPA recommendations was the lowest in 2001, peaked in 2004 or 2009 and then declined in 2015. However, such inverted U-shaped trend was found to be significant only among males, those aged $50-59$ years, inhabitants of urban areas, formerly married, nonBuddhists, the unemployed, and those who did not attend school or had secondary education.

The prevalence of Thai adults who met the MVPA recommendations for additional health benefits was 74.3\% (95\% CI: 73.9, 74. 7) in 2015 (Additional File 1). For the overall sample and for most sociodemographic groups, the sample prevalence was lowest in 2001, rose to its highest point in 2004 and 2009, and then declined in 2015. However, this inverted U-shaped trend was found to be significant only among those who were aged $50-59$ years, formerly married, and with unspecified level of education. 


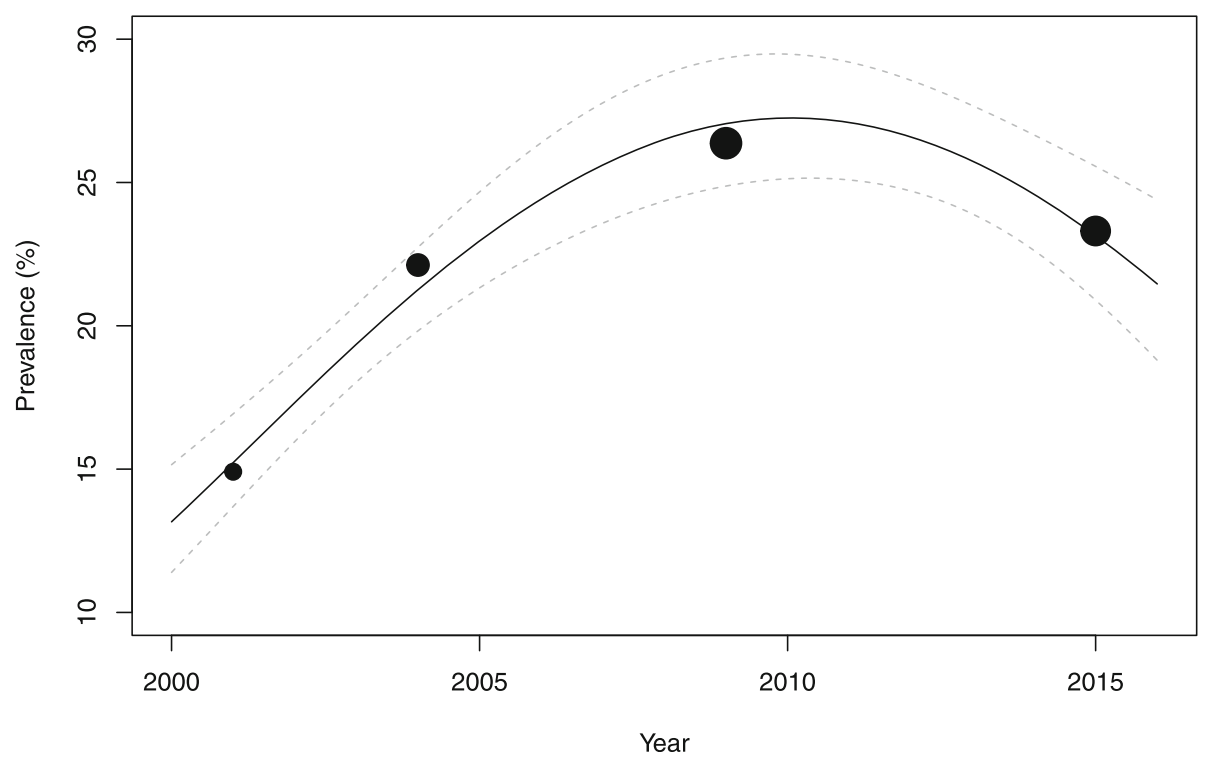

Fig. 1 The trend in prevalence of meeting the 24-h movement guidelines among females. The size of the circles is proportional to the precision of each survey year's estimate of the prevalence

\section{Associations of sociodemographic characteristics with meeting the MVPA recommendations}

In all survey years, older age groups (i.e., 40-49 years and 50-59 years) had higher odds of meeting the MVPA recommendations, compared to the youngest adult group (18-29 years; Table 3). Rural residents had 95, 45, 19 and 19\% higher odds of meeting the recommendation than those who lived in urban areas in 2001, 2004, 2009, and 2015 , respectively ( $p$-value for linear trend $=0.042$ ).
Those who lived in Bangkok had the lowest odds, while the participants from the North region had the highest odds of meeting the recommendations (in all years except in 2001). An inverted U-shaped trend was found for the odds ratios for the North region, where the odds rose from 2001 to 2009 and then dropped in 2015 ( $p=$ $0.025)$. In most survey years, significantly higher odds of meeting the MVPA recommendations were also found for those who were married (compared to those who

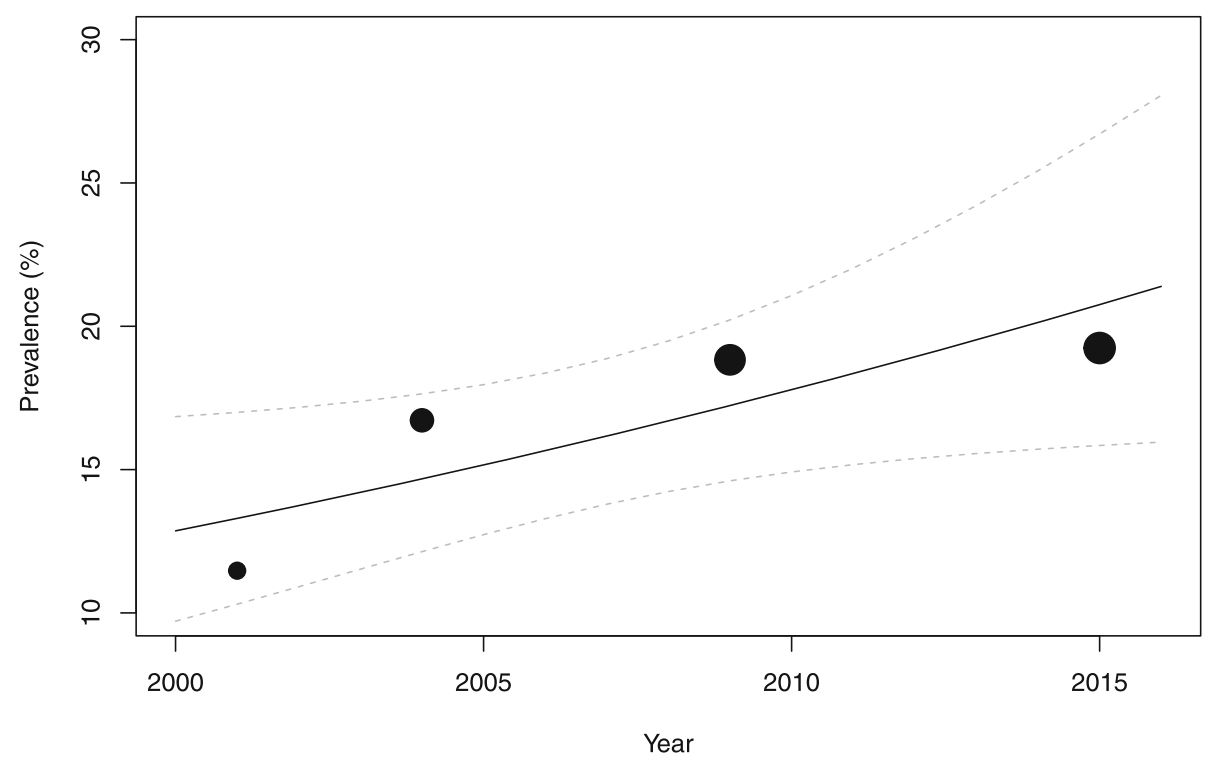

Fig. 2 The trend in prevalence of meeting the 24-movement guidelines among males. The size of the circles is proportional to the precision of each survey year's estimate of the prevalence 
Table 2 Meeting the overall guidelines: population prevalence and associations with sociodemographic variables

\begin{tabular}{|c|c|c|c|c|c|c|c|c|c|c|}
\hline \multirow{2}{*}{$\begin{array}{l}\text { Sociodemographic } \\
\text { variable }\end{array}$} & \multicolumn{5}{|c|}{ Percentage (95\% Cl) } & \multicolumn{5}{|c|}{ Adjusted OR (95\% Cl) } \\
\hline & 2001 & 2004 & 2009 & 2015 & $p$-value & 2001 & 2004 & 2009 & 2015 & $\begin{array}{l}p- \\
\text { value }\end{array}$ \\
\hline Total $(n)$ & $\begin{array}{l}13.2(12.9- \\
13.5)\end{array}$ & $\begin{array}{l}19.4(19.0- \\
19.8)\end{array}$ & $\begin{array}{l}22.7(22.3- \\
23.0)\end{array}$ & $\begin{array}{l}21.3(20.9- \\
21.7)\end{array}$ & $0.093^{*}$ & & & & & \\
\hline \multicolumn{11}{|l|}{ Sex } \\
\hline Male & $\begin{array}{l}11.5(11.0- \\
11.9)\end{array}$ & $\begin{array}{l}16.7(16.2- \\
17.3)\end{array}$ & $\begin{array}{l}18.8(18.3- \\
19.3)\end{array}$ & $\begin{array}{l}19.2(18.7- \\
19.8)\end{array}$ & $0.033^{*}$ & Ref & & & & \\
\hline Female & $\begin{array}{l}14.9(14.4- \\
15.4)\end{array}$ & $\begin{array}{l}22.1(21.5- \\
22.7)\end{array}$ & $\begin{array}{l}26.4(25.8- \\
26.9)\end{array}$ & $\begin{array}{l}23.3(22.8- \\
23.9)\end{array}$ & $\begin{array}{l}< \\
0.001^{* *}\end{array}$ & $\begin{array}{l}1.53(1.44- \\
1.63)\end{array}$ & $\begin{array}{l}1.60(1.51- \\
1.69)\end{array}$ & $\begin{array}{l}1.73(1.65- \\
1.81)\end{array}$ & $\begin{array}{l}1.43(1.36- \\
1.50)\end{array}$ & $0.748^{*}$ \\
\hline \multicolumn{11}{|l|}{ Age } \\
\hline $18-29$ & $\begin{array}{l}11.4(10.9- \\
12.0)\end{array}$ & $\begin{array}{l}17.9(17.2- \\
18.6)\end{array}$ & $\begin{array}{l}20.0(19.3- \\
20.6)\end{array}$ & $\begin{array}{l}17.9(17.3- \\
18.6)\end{array}$ & $0.219^{*}$ & Ref & & & & \\
\hline $30-39$ & $\begin{array}{l}14.7(14.0- \\
15.4)\end{array}$ & $\begin{array}{l}20.8(20.0- \\
21.6)\end{array}$ & $\begin{array}{l}25.7(24.9- \\
26.4)\end{array}$ & $\begin{array}{l}23.7(22.9- \\
24.5)\end{array}$ & $\begin{array}{l}< \\
0.001^{* *}\end{array}$ & $\begin{array}{l}1.00(0.92- \\
1.09)\end{array}$ & $\begin{array}{l}0.90(0.84- \\
0.97)\end{array}$ & $\begin{array}{l}1.14(1.07- \\
1.22)\end{array}$ & $\begin{array}{l}1.11(1.03- \\
1.18)\end{array}$ & $0.497^{*}$ \\
\hline $40-49$ & $\begin{array}{l}14.7(14.0- \\
15.5)\end{array}$ & $\begin{array}{l}20.4(19.5- \\
21.2)\end{array}$ & $\begin{array}{l}23.1(22.3- \\
23.9)\end{array}$ & $\begin{array}{l}23.2(22.4- \\
23.9)\end{array}$ & $0.034^{*}$ & $\begin{array}{l}0.94(0.86- \\
1.03)\end{array}$ & $\begin{array}{l}0.86(0.79- \\
0.93)\end{array}$ & $\begin{array}{l}0.96(0.90- \\
1.03)\end{array}$ & $\begin{array}{l}1.04(0.97- \\
1.12)\end{array}$ & $0.576^{*}$ \\
\hline $50-59$ & $\begin{array}{l}12.4(11.6- \\
13.3)\end{array}$ & $\begin{array}{l}18.9(17.9- \\
19.9)\end{array}$ & $\begin{array}{l}22.1(21.2- \\
23.0)\end{array}$ & $\begin{array}{l}20.7(19.9- \\
21.5)\end{array}$ & $0.100^{*}$ & $\begin{array}{l}0.82(0.74- \\
0.91)\end{array}$ & $\begin{array}{l}0.83(0.75- \\
0.90)\end{array}$ & $\begin{array}{l}0.98(0.91- \\
1.06)\end{array}$ & $\begin{array}{l}0.95(0.88- \\
1.03)\end{array}$ & $0.526^{*}$ \\
\hline \multicolumn{11}{|l|}{ Household area } \\
\hline Urban & $9.8(9.3-10.3)$ & $\begin{array}{l}17.8(17.2- \\
18.5)\end{array}$ & $\begin{array}{l}21.6(20.9- \\
22.2)\end{array}$ & $\begin{array}{l}21.4(20.8- \\
21.9)\end{array}$ & $0.053^{*}$ & Ref & & & & \\
\hline Rural & $\begin{array}{l}14.9(14.4- \\
15.3)\end{array}$ & $\begin{array}{l}20.2(19.7- \\
20.7)\end{array}$ & $\begin{array}{l}23.2(22.7- \\
23.6)\end{array}$ & $\begin{array}{l}21.3(20.7- \\
21.8)\end{array}$ & $\begin{array}{l}< \\
0.001^{* *}\end{array}$ & $\begin{array}{l}1.19(1.10- \\
1.30)\end{array}$ & $\begin{array}{l}0.95(0.89- \\
1.02)\end{array}$ & $\begin{array}{l}0.95(0.90- \\
1.00)\end{array}$ & $\begin{array}{l}0.94(0.89- \\
0.98)\end{array}$ & $0.471^{*}$ \\
\hline \multicolumn{11}{|l|}{ Region } \\
\hline Bangkok & $8.4(7.6-9.2)$ & $\begin{array}{l}15.1(14.1- \\
16.1)\end{array}$ & $\begin{array}{l}17.0(16.0- \\
18.1)\end{array}$ & $\begin{array}{l}19.8(18.9- \\
20.8)\end{array}$ & $0.011^{*}$ & Ref & & & & \\
\hline Central & $\begin{array}{l}11.3(10.7- \\
12.0)\end{array}$ & $\begin{array}{l}18.4(17.6- \\
19.2)\end{array}$ & $\begin{array}{l}22.7(21.9- \\
23.5)\end{array}$ & $\begin{array}{l}21.3(20.6- \\
21.9)\end{array}$ & $\begin{array}{l}< \\
0.001^{* *}\end{array}$ & $\begin{array}{l}1.10(0.96- \\
1.26)\end{array}$ & $\begin{array}{l}1.20(1.08- \\
1.33)\end{array}$ & $\begin{array}{l}1.52(1.39- \\
1.68)\end{array}$ & $\begin{array}{l}1.09(1.01- \\
1.18)\end{array}$ & $0.951^{*}$ \\
\hline North & $\begin{array}{l}16.3(15.5- \\
17.2)\end{array}$ & $\begin{array}{l}23.8(22.8- \\
24.8)\end{array}$ & $\begin{array}{l}24.4(23.5- \\
25.3)\end{array}$ & $\begin{array}{l}22.9(21.9- \\
23.8)\end{array}$ & $0.288^{*}$ & $\begin{array}{l}1.50(1.30- \\
1.73)\end{array}$ & $\begin{array}{l}1.67(1.49- \\
1.86)\end{array}$ & $\begin{array}{l}1.69(1.52- \\
1.87)\end{array}$ & $\begin{array}{l}1.25(1.14- \\
1.36)\end{array}$ & $0.454^{*}$ \\
\hline North-East & $\begin{array}{l}15.0(14.4- \\
15.7)\end{array}$ & $\begin{array}{l}20.7(19.9- \\
21.4)\end{array}$ & $\begin{array}{l}24.5(23.8- \\
25.1)\end{array}$ & $\begin{array}{l}21.7(20.9- \\
22.4)\end{array}$ & $\begin{array}{l}< \\
0.001^{* *}\end{array}$ & $\begin{array}{l}1.38(1.21- \\
1.59)\end{array}$ & $\begin{array}{l}1.34(1.21- \\
1.49)\end{array}$ & $\begin{array}{l}1.75(1.59- \\
1.93)\end{array}$ & $\begin{array}{l}1.19(1.10- \\
1.29)\end{array}$ & $0.707^{*}$ \\
\hline South & $\begin{array}{l}11.6(10.7- \\
12.6)\end{array}$ & $\begin{array}{l}16.4(15.4- \\
17.5)\end{array}$ & $\begin{array}{l}20.2(19.2- \\
21.2)\end{array}$ & $\begin{array}{l}20.6(19.6- \\
21.6)\end{array}$ & $0.010^{*}$ & $\begin{array}{l}1.02(0.87- \\
1.20)\end{array}$ & $\begin{array}{l}1.03(0.91- \\
1.17)\end{array}$ & $\begin{array}{l}1.30(1.16- \\
1.45)\end{array}$ & $\begin{array}{l}1.05(0.96- \\
1.16)\end{array}$ & $0.875^{*}$ \\
\hline \multicolumn{11}{|l|}{ Marital status } \\
\hline Never married & $8.2(7.7-8.8)$ & $\begin{array}{l}12.9(12.2- \\
13.6)\end{array}$ & $\begin{array}{l}17.0(16.2- \\
17.7)\end{array}$ & $\begin{array}{l}16.6(16.0- \\
17.3)\end{array}$ & $\begin{array}{l}<.001^{* *} \\
\end{array}$ & Ref & & & & \\
\hline Married & $\begin{array}{l}15.0(14.6- \\
15.5)\end{array}$ & $\begin{array}{l}21.9(21.4- \\
22.4)\end{array}$ & $\begin{array}{l}24.4(23.9- \\
24.8)\end{array}$ & $\begin{array}{l}23.3(22.8- \\
23.7)\end{array}$ & $0.113^{*}$ & $\begin{array}{l}1.49(1.36- \\
1.64)\end{array}$ & $\begin{array}{l}1.65(1.53- \\
1.79)\end{array}$ & $\begin{array}{l}1.38(1.30- \\
1.47)\end{array}$ & $\begin{array}{l}1.34(1.26- \\
1.43)\end{array}$ & $0.509^{*}$ \\
\hline $\begin{array}{l}\text { Formerly } \\
\text { married }\end{array}$ & $\begin{array}{l}12.3(11.0- \\
13.7)\end{array}$ & $\begin{array}{l}17.5(15.9- \\
19.1)\end{array}$ & $\begin{array}{l}24.1(22.6- \\
25.6)\end{array}$ & $\begin{array}{l}21.8(20.5- \\
23.2)\end{array}$ & $\begin{array}{l}< \\
0.001^{* *}\end{array}$ & $\begin{array}{l}1.17(0.99- \\
1.37)\end{array}$ & $\begin{array}{l}1.25(1.09- \\
1.43)\end{array}$ & $\begin{array}{l}1.31(1.18- \\
1.46)\end{array}$ & $\begin{array}{l}1.23(1.12- \\
1.36)\end{array}$ & $0.905^{*}$ \\
\hline \multicolumn{11}{|l|}{ Religion } \\
\hline Buddhist & $\begin{array}{l}13.1(12.8- \\
13.5)\end{array}$ & $\begin{array}{l}19.6(19.2- \\
20.0)\end{array}$ & $\begin{array}{l}22.7(22.3- \\
23.1)\end{array}$ & $\begin{array}{l}21.3(20.9- \\
21.7)\end{array}$ & $0.103^{*}$ & Ref & & & & \\
\hline Non-Buddhist & $\begin{array}{l}14.3(12.7- \\
15.9)\end{array}$ & $\begin{array}{l}16.5(14.8- \\
18.2)\end{array}$ & $\begin{array}{l}21.6(19.9- \\
23.3)\end{array}$ & $\begin{array}{l}21.6(19.8- \\
23.3)\end{array}$ & $0.003^{*}$ & $\begin{array}{l}1.16(1.00- \\
1.35)\end{array}$ & $\begin{array}{l}1.00(0.86- \\
1.15)\end{array}$ & $\begin{array}{l}1.12(0.99- \\
1.25)\end{array}$ & $\begin{array}{l}1.11(0.99- \\
1.24)\end{array}$ & $0.976^{*}$ \\
\hline \multicolumn{11}{|l|}{ Employment status } \\
\hline Employed & $\begin{array}{l}15.0(14.6- \\
15.4)\end{array}$ & $\begin{array}{l}21.8(21.3- \\
22.2)\end{array}$ & $\begin{array}{l}25.0(24.6- \\
25.4)\end{array}$ & $\begin{array}{l}24.0(23.6- \\
24.4)\end{array}$ & $0.079^{*}$ & Ref & & & & \\
\hline Unemployed & $4.8(4.3-5.3)$ & $8.3(7.6-9.0)$ & $\begin{array}{l}11.1(10.4- \\
11.8)\end{array}$ & $10.0(9.4-10.6)$ & $\begin{array}{l}< \\
0.001^{* *}\end{array}$ & $\begin{array}{l}0.28(0.25- \\
0.32)\end{array}$ & $\begin{array}{l}0.31(0.28- \\
0.34)\end{array}$ & $\begin{array}{l}0.34(0.31- \\
0.37)\end{array}$ & $\begin{array}{l}0.34(0.31- \\
0.36)\end{array}$ & $0.571^{*}$ \\
\hline \multicolumn{11}{|c|}{ Highest education level } \\
\hline None & $\begin{array}{l}17.1(15.0- \\
19.2)\end{array}$ & $\begin{array}{l}17.3(15.1- \\
19.4)\end{array}$ & $\begin{array}{l}20.3(18.6- \\
22.1)\end{array}$ & $\begin{array}{l}19.8(18.6- \\
20.9)\end{array}$ & $0.070^{*}$ & Ref & & & & \\
\hline Primary & $\begin{array}{l}15.4(14.9- \\
15.8)\end{array}$ & $\begin{array}{l}20.7(20.1- \\
21.2)\end{array}$ & $\begin{array}{l}22.8(22.2- \\
23.3)\end{array}$ & $\begin{array}{l}21.8(21.2- \\
22.4)\end{array}$ & $0.112^{*}$ & $\begin{array}{l}0.84(0.71- \\
0.99)\end{array}$ & $\begin{array}{l}1.16(0.99- \\
1.37)\end{array}$ & $\begin{array}{l}1.01(0.91- \\
1.14)\end{array}$ & $\begin{array}{l}1.06(0.98- \\
1.16)\end{array}$ & $0.701^{*}$ \\
\hline Secondary & $9.8(9.3-10.4)$ & $17.7(17.0-$ & $22.2(21.5-$ & $21.5(20.8-$ & $<$ & 0.68 (0.57- & $1.21(1.03-$ & $1.15(1.02-$ & $1.13(1.04-$ & $0.347^{*}$ \\
\hline
\end{tabular}


Table 2 Meeting the overall guidelines: population prevalence and associations with sociodemographic variables (Continued)

\begin{tabular}{|c|c|c|c|c|c|c|c|c|c|c|}
\hline \multirow{2}{*}{$\begin{array}{l}\text { Sociodemographic } \\
\text { variable }\end{array}$} & \multicolumn{5}{|c|}{ Percentage $(95 \% \mathrm{Cl})$} & \multicolumn{5}{|c|}{ Adjusted OR (95\% Cl) } \\
\hline & 2001 & 2004 & 2009 & 2015 & $p$-value & 2001 & 2004 & 2009 & 2015 & $\begin{array}{l}p- \\
\text { value }\end{array}$ \\
\hline & & 18.5) & 22.9) & 22.2) & $0.001^{* *}$ & $0.81)$ & 1.44) & 1.29) & 1.23) & \\
\hline $\begin{array}{l}\text { Higher } \\
\text { education }\end{array}$ & $7.9(7.1-8.7)$ & $\begin{array}{l}18.5(17.4- \\
19.6)\end{array}$ & $\begin{array}{l}23.7(22.7- \\
24.6)\end{array}$ & $\begin{array}{l}20.9(20.1- \\
21.6)\end{array}$ & $\begin{array}{l}< \\
0.001^{* *}\end{array}$ & $\begin{array}{l}0.47(0.39- \\
0.58)\end{array}$ & $\begin{array}{l}1.13(0.95- \\
1.35)\end{array}$ & $\begin{array}{l}1.26(1.12- \\
1.43)\end{array}$ & $\begin{array}{l}1.15(1.05- \\
1.26)\end{array}$ & $0.046^{* *}$ \\
\hline Unspecified & $14.8(7.1-22.5)$ & $14.3(8.1-20.5)$ & $\begin{array}{l}32.3(23.7- \\
40.9)\end{array}$ & $\begin{array}{l}26.5(20.9- \\
32.0)\end{array}$ & $0.120^{*}$ & $\begin{array}{l}1.10(0.55- \\
2.02)\end{array}$ & $\begin{array}{l}0.92(0.52- \\
1.54)\end{array}$ & $\begin{array}{l}1.82(1.19- \\
2.74)\end{array}$ & $\begin{array}{l}1.31(0.97- \\
1.76)\end{array}$ & $0.639^{*}$ \\
\hline
\end{tabular}

$\mathrm{Cl}$ Confidence interval, OR Odds ratio adjusted for all other variables in the table, Ref Reference group

${ }^{*}=$ linear model; ${ }^{* *}=$ quadratic model

have never been married), non-Buddhists (compared to Buddhists), and employed (compared to unemployed). Regarding level of education, the lowest odds of meeting the MVPA recommendations were found for those with no formal education.

In all survey years, males had higher odds of meeting the MVPA recommendations for additional health benefits, compared to females (Additional File 1). Older ages (30-59 years) also had higher odds of meeting the recommendations than the youngest adult group (18-29 years). The odds of meeting the recommendations were $2.09,1.54,1.35$, and 1.27 times higher among rural residents, compared with those who lived in urban areas in $2001,2004,2009$, and 2015, respectively ( $p$-value for linear trend $=0.039$ ). Thai adults who lived in Bangkok had the lowest odds of meeting the recommendations, while those who lived in the North region had the highest odds in all survey years except in 2001. In all survey years, higher odds of meeting the recommendations were also found to be significant for those who were non-Buddhists (compared to Buddhists), and employed (compared to unemployed). For the education level, Thai adults who did not attend school had the lowest odds of meeting the recommendations in all survey years except in 2001. An inverted U-shaped trend was found for the odds ratios for those who had higher education, where the odds increased from 2001 to 2009 and then declined in $2015(p=0.004)$.

\section{Prevalence of meeting the SB recommendation}

In 2015, the prevalence of Thai adults who met the SB recommendation was $44.6 \%$ (95\% CI: 44.1, 45.0). A significant linear increase was found for the whole sample, males, all age groups above 30 years of age, those who lived in urban or rural areas, residents of all regions except the North, those who were married and those who have never been married, Buddhists, employed, and those with primary and secondary education. The sample prevalence of adults aged 18-29 years, unemployed, and those with higher education was found to be lowest in 2001, rose to its highest in 2009, and then declined in 2015 ( $p$-value for inverted $U$-shaped trend $<0.05$ ).

\section{Associations of sociodemographic characteristics with meeting the SB recommendation}

In 2001, 2004, 2009, and 2015, females had 23, 47, 85, and $44 \%$, respectively, higher odds of meeting the SB recommendation than males (Table 4). The youngest adult group (18-29 years) had the highest odds of meeting the recommendation compared to the remaining age groups. Those who lived in urban areas had higher odds of meeting the recommendation than those who lived in rural areas in all survey years except in 2001. Significantly higher odds of meeting the SB recommendation were also found among those who were married or formerly married (compared to those who have never been married), and employed (compared to unemployed). For those who had higher education, the odds of meeting the SB recommendation rose from 2001 to 2009 and dropped in 2015 (quadratic trend $p=$ $0.048)$.

\section{Prevalence of meeting the sleep recommendation}

In 2015, the prevalence of Thai adults who met the sleep recommendation was $56.4 \%$ (95\% CI: 56.0, 56.9). For the overall sample and for most sociodemographic groups, the sample prevalence of adults meeting the sleep recommendation was lowest in 2001, rose to its highest in 2009, and then declined in 2015. However, an inverted $\mathrm{U}$-shaped trend was found to be significant only in the overall sample, among Buddhists, and among those who had secondary or higher education.

\section{Associations of sociodemographic characteristics with meeting the sleep recommendation}

Females had 28, 25, 32, and 35\% higher odds of meeting the sleep recommendation than males in 2001, 2004, 2009, and 2015, respectively (Table 5). In most survey years, significantly higher odds of meeting the recommendation were found for the youngest adult group (compared to those aged 50-59 years), inhabitants of the North-East region (compared to those from Bangkok), married (compared to those who have never been married), and employed (compared to unemployed). Thai adults without formal education had the lowest odds of meeting the sleep recommendation. 
Table 3 Meeting the moderate-to-vigorous physical activity guideline: population prevalence and associations with sociodemographic variables

\begin{tabular}{|c|c|c|c|c|c|c|c|c|c|c|}
\hline \multirow{2}{*}{$\begin{array}{l}\text { Sociodemographic } \\
\text { variable }\end{array}$} & \multicolumn{5}{|c|}{ Percentage $(95 \% \mathrm{Cl})$} & \multicolumn{5}{|c|}{ Adjusted OR (95\% Cl) } \\
\hline & 2001 & 2004 & 2009 & 2015 & $p$-value & 2001 & 2004 & 2009 & 2015 & $\begin{array}{l}p- \\
\text { value }\end{array}$ \\
\hline Total $(n)$ & $\begin{array}{l}73.2(72.8- \\
73.7)\end{array}$ & $\begin{array}{l}88.1(87.7- \\
88.4)\end{array}$ & $\begin{array}{l}87.6(87.3- \\
87.9)\end{array}$ & $\begin{array}{l}81.7(81.3- \\
82.1)\end{array}$ & $0.078^{* *}$ & & & & & \\
\hline \multicolumn{11}{|l|}{ Sex } \\
\hline Male & $\begin{array}{l}73.7(73.1- \\
74.4)\end{array}$ & $\begin{array}{l}89.2(88.7- \\
89.6)\end{array}$ & $\begin{array}{l}89.6(89.2- \\
90.0)\end{array}$ & $\begin{array}{l}83.6(83.1- \\
84.0)\end{array}$ & $0.024^{* *}$ & Ref & & & & \\
\hline Female & $\begin{array}{l}72.7(72.1- \\
73.3)\end{array}$ & $\begin{array}{l}87.0(86.5- \\
87.5)\end{array}$ & $\begin{array}{l}85.6(85.2- \\
86.1)\end{array}$ & $\begin{array}{l}79.9(79.4- \\
80.4)\end{array}$ & $0.151^{* *}$ & $\begin{array}{l}1.01(0.96- \\
1.06)\end{array}$ & $\begin{array}{l}0.96(0.90- \\
1.03)\end{array}$ & $\begin{array}{l}0.74(0.70- \\
0.78)\end{array}$ & $\begin{array}{l}0.82(0.78- \\
0.86)\end{array}$ & $0.269^{*}$ \\
\hline \multicolumn{11}{|l|}{ Age } \\
\hline $18-29$ & $\begin{array}{l}67.9(67.1- \\
68.7)\end{array}$ & $\begin{array}{l}85.0(84.4- \\
85.6)\end{array}$ & $\begin{array}{l}83.9(83.2- \\
84.5)\end{array}$ & $\begin{array}{l}77.5(76.8- \\
78.2)\end{array}$ & $0.127^{* *}$ & Ref & & & & \\
\hline $30-39$ & $\begin{array}{l}73.4(72.6- \\
74.3)\end{array}$ & $\begin{array}{l}88.4(87.8- \\
89.1)\end{array}$ & $\begin{array}{l}86.7(86.1- \\
87.3)\end{array}$ & $\begin{array}{l}80.2(79.5- \\
81.0)\end{array}$ & $0.171^{* *}$ & $\begin{array}{l}1.18(1.10- \\
1.26)\end{array}$ & $\begin{array}{l}1.04(0.95- \\
1.13)\end{array}$ & $\begin{array}{l}1.19(1.10- \\
1.29)\end{array}$ & $\begin{array}{l}1.06(0.99- \\
1.13)\end{array}$ & $0.811^{*}$ \\
\hline $40-49$ & $\begin{array}{l}77.3(76.4- \\
78.2)\end{array}$ & $\begin{array}{l}91.0(90.4- \\
91.6)\end{array}$ & $\begin{array}{l}90.6(90.1- \\
91.2)\end{array}$ & $\begin{array}{l}84.3(83.7- \\
85.0)\end{array}$ & $0.054^{* *}$ & $\begin{array}{l}1.36(1.26- \\
1.47)\end{array}$ & $\begin{array}{l}1.38(1.25- \\
1.54)\end{array}$ & $\begin{array}{l}1.70(1.55- \\
1.86)\end{array}$ & $\begin{array}{l}1.24(1.15- \\
1.33)\end{array}$ & $0.818^{*}$ \\
\hline $50-59$ & $\begin{array}{l}79.5(78.4- \\
80.5)\end{array}$ & $\begin{array}{l}89.7(88.9- \\
90.5)\end{array}$ & $\begin{array}{l}90.4(89.8- \\
91.0)\end{array}$ & $\begin{array}{l}85.5(84.8- \\
86.1)\end{array}$ & $0.005^{* *}$ & $\begin{array}{l}1.48(1.36- \\
1.62)\end{array}$ & $\begin{array}{l}1.23(1.10- \\
1.39)\end{array}$ & $\begin{array}{l}1.71(1.55- \\
1.89)\end{array}$ & $\begin{array}{l}1.31(1.21- \\
1.42)\end{array}$ & $0.887^{*}$ \\
\hline \multicolumn{11}{|l|}{ Household area } \\
\hline Urban & $\begin{array}{l}57.6(56.8- \\
58.5)\end{array}$ & $\begin{array}{l}81.2(80.5- \\
81.8)\end{array}$ & $\begin{array}{l}82.4(81.8- \\
83.0)\end{array}$ & $\begin{array}{l}77.3(76.7- \\
77.8)\end{array}$ & $0.034^{* *}$ & Ref & & & & \\
\hline Rural & $\begin{array}{l}80.8(80.3- \\
81.3)\end{array}$ & $\begin{array}{l}91.6(91.3- \\
92.0)\end{array}$ & $\begin{array}{l}90.0(89.7- \\
90.3)\end{array}$ & $\begin{array}{l}85.6(85.2- \\
86.1)\end{array}$ & $0.237^{* *}$ & $\begin{array}{l}1.95(1.84- \\
2.07)\end{array}$ & $\begin{array}{l}1.45(1.33- \\
1.57)\end{array}$ & $\begin{array}{l}1.19(1.10- \\
1.28)\end{array}$ & $\begin{array}{l}1.19(1.12- \\
1.26)\end{array}$ & $0.042^{*}$ \\
\hline \multicolumn{11}{|l|}{ Region } \\
\hline Bangkok & $\begin{array}{l}49.9(48.4- \\
51.3)\end{array}$ & $\begin{array}{l}73.0(71.7- \\
74.2)\end{array}$ & $\begin{array}{l}73.3(72.1- \\
74.6)\end{array}$ & $\begin{array}{l}67.7(66.6- \\
68.8)\end{array}$ & $0.069^{* *}$ & Ref & & & & \\
\hline Central & $\begin{array}{l}67.5(66.6- \\
68.5)\end{array}$ & $\begin{array}{l}86.7(86.0- \\
87.4)\end{array}$ & $\begin{array}{l}84.0(83.3- \\
84.7)\end{array}$ & $\begin{array}{l}78.6(77.9- \\
79.3)\end{array}$ & $0.278^{* *}$ & $\begin{array}{l}1.21(1.11- \\
1.31)\end{array}$ & $\begin{array}{l}1.80(1.62- \\
1.99)\end{array}$ & $\begin{array}{l}1.76(1.61- \\
1.94)\end{array}$ & $\begin{array}{l}1.58(1.47- \\
1.70)\end{array}$ & $0.497^{*}$ \\
\hline North & $\begin{array}{l}78.7 \text { (77.8- } \\
79.7)\end{array}$ & $\begin{array}{l}93.1(92.5- \\
93.7)\end{array}$ & $\begin{array}{l}92.7(92.2- \\
93.3)\end{array}$ & $\begin{array}{l}89.3(88.6- \\
90.0)\end{array}$ & $0.107^{* *}$ & $\begin{array}{l}1.82(1.66- \\
1.99)\end{array}$ & $\begin{array}{l}3.54(3.11- \\
4.03)\end{array}$ & $\begin{array}{l}4.12(3.66- \\
4.64)\end{array}$ & $\begin{array}{l}3.46(3.14- \\
3.81)\end{array}$ & $0.025^{* *}$ \\
\hline North-East & $\begin{array}{l}82.0(81.3- \\
82.7)\end{array}$ & $\begin{array}{l}91.7(91.2- \\
92.2)\end{array}$ & $\begin{array}{l}90.7 \text { (90.3- } \\
91.2)\end{array}$ & $\begin{array}{l}85.9(85.3- \\
86.5)\end{array}$ & $0.122^{* *}$ & $\begin{array}{l}2.21(2.02- \\
2.41)\end{array}$ & $\begin{array}{l}2.73(2.44- \\
3.06)\end{array}$ & $\begin{array}{l}3.20(2.88- \\
3.55)\end{array}$ & $\begin{array}{l}2.48(2.29- \\
2.70)\end{array}$ & $0.708^{*}$ \\
\hline South & $\begin{array}{l}74.9 \text { (73.6- } \\
76.1)\end{array}$ & $\begin{array}{l}90.9 \text { (90.0- } \\
91.7)\end{array}$ & $\begin{array}{l}90.3(89.6- \\
91.0)\end{array}$ & $\begin{array}{l}87.3(86.5- \\
88.2)\end{array}$ & $0.140^{* *}$ & $\begin{array}{l}1.49(1.35- \\
1.65)\end{array}$ & $\begin{array}{l}2.49(2.17- \\
2.87)\end{array}$ & $\begin{array}{l}2.92(2.59- \\
3.30)\end{array}$ & $\begin{array}{l}2.77(2.51- \\
3.06)\end{array}$ & $0.080^{*}$ \\
\hline \multicolumn{11}{|l|}{ Marital status } \\
\hline Never married & $\begin{array}{l}65.4(64.5- \\
66.4)\end{array}$ & $\begin{array}{l}82.6(81.8- \\
83.4)\end{array}$ & $\begin{array}{l}82.7(82.0- \\
83.4)\end{array}$ & $\begin{array}{l}76.9 \text { (76.1- } \\
77.6)\end{array}$ & $0.056^{* *}$ & Ref & & & & \\
\hline Married & $\begin{array}{l}76.0(75.4- \\
76.5)\end{array}$ & $\begin{array}{l}90.4(89.7- \\
90.4)\end{array}$ & $\begin{array}{l}89.2(88.8- \\
89.5)\end{array}$ & $\begin{array}{l}83.7(83.3- \\
84.1)\end{array}$ & $0.119^{* *}$ & $\begin{array}{l}0.97(0.91- \\
1.04)\end{array}$ & $\begin{array}{l}1.24(1.14- \\
1.34)\end{array}$ & $\begin{array}{l}1.22(1.13- \\
1.31)\end{array}$ & $\begin{array}{l}1.13(1.06- \\
1.20)\end{array}$ & $0.664^{*}$ \\
\hline $\begin{array}{l}\text { Formerly } \\
\text { married }\end{array}$ & $\begin{array}{l}73.1(71.3- \\
75.0)\end{array}$ & $\begin{array}{l}86.9(85.8- \\
88.3)\end{array}$ & $\begin{array}{l}87.5(86.3- \\
88.6)\end{array}$ & $\begin{array}{l}82.5(81.2- \\
83.7)\end{array}$ & $0.024^{* *}$ & $\begin{array}{l}0.82(0.73- \\
0.92)\end{array}$ & $\begin{array}{l}0.95(0.81- \\
1.11)\end{array}$ & $\begin{array}{l}1.09(0.96- \\
1.24)\end{array}$ & $\begin{array}{l}1.04(0.93- \\
1.15)\end{array}$ & $0.477^{*}$ \\
\hline \multicolumn{11}{|l|}{ Religion } \\
\hline Buddhist & $\begin{array}{l}72.9(72.5- \\
73.4)\end{array}$ & $\begin{array}{l}88.0(87.6- \\
88.3)\end{array}$ & $\begin{array}{l}87.4(87.1- \\
87.7)\end{array}$ & $\begin{array}{l}81.5(81.1- \\
81.8)\end{array}$ & $0.082^{* *}$ & Ref & & & & \\
\hline Non-Buddhist & $\begin{array}{l}78.8(77.0- \\
80.7)\end{array}$ & $\begin{array}{l}90.4(89.0- \\
91.7)\end{array}$ & $\begin{array}{l}90.7 \text { (89.5- } \\
91.9)\end{array}$ & $\begin{array}{l}86.3(84.9- \\
87.8)\end{array}$ & $0.020^{* *}$ & $\begin{array}{l}1.44(1.27- \\
1.63)\end{array}$ & $\begin{array}{l}1.2(1.01- \\
1.45)\end{array}$ & $\begin{array}{l}1.49(1.28- \\
1.75)\end{array}$ & $\begin{array}{l}1.25(1.09- \\
1.43)\end{array}$ & $0.831^{*}$ \\
\hline \multicolumn{11}{|l|}{ Employment status } \\
\hline Employed & $\begin{array}{l}74.8(74.3- \\
75.3)\end{array}$ & $\begin{array}{l}90.5(90.2- \\
90.8)\end{array}$ & $\begin{array}{l}89.1(88.8- \\
89.4)\end{array}$ & $\begin{array}{l}83.3(82.9- \\
83.7)\end{array}$ & $0.168^{* *}$ & Ref & & & & \\
\hline Unemployed & $\begin{array}{l}65.6(64.5- \\
66.8)\end{array}$ & $\begin{array}{l}76.6(75.6- \\
77.6)\end{array}$ & $\begin{array}{l}80.1(79.3- \\
81.0)\end{array}$ & $\begin{array}{l}75.0(74.1- \\
75.9)\end{array}$ & $\begin{array}{l}< \\
0.001^{* *}\end{array}$ & $\begin{array}{l}0.74(0.69- \\
0.78)\end{array}$ & $\begin{array}{l}0.41(0.38- \\
0.44)\end{array}$ & $\begin{array}{l}0.59(0.55- \\
0.63)\end{array}$ & $\begin{array}{l}0.63(0.59- \\
0.66)\end{array}$ & $0.947^{*}$ \\
\hline
\end{tabular}


Table 3 Meeting the moderate-to-vigorous physical activity guideline: population prevalence and associations with sociodemographic variables (Continued)

\begin{tabular}{|c|c|c|c|c|c|c|c|c|c|c|}
\hline \multirow{2}{*}{$\begin{array}{l}\text { Sociodemographic } \\
\text { variable }\end{array}$} & \multicolumn{5}{|c|}{ Percentage (95\% Cl) } & \multicolumn{5}{|c|}{ Adjusted OR (95\% Cl) } \\
\hline & 2001 & 2004 & 2009 & 2015 & $p$-value & 2001 & 2004 & 2009 & 2015 & $\begin{array}{l}p- \\
\text { value }\end{array}$ \\
\hline \multicolumn{11}{|c|}{ Highest education level } \\
\hline None & $\begin{array}{l}79.9(77.6- \\
82.2)\end{array}$ & $\begin{array}{l}84.4(82.4- \\
86.5)\end{array}$ & $\begin{array}{l}84.6(83.1- \\
86.2)\end{array}$ & $\begin{array}{l}78.6(77.4- \\
79.8)\end{array}$ & $\begin{array}{l}< \\
0.001^{* *}\end{array}$ & Ref & & & & \\
\hline Primary & $\begin{array}{l}80.7(80.2- \\
81.2)\end{array}$ & $\begin{array}{l}91.0(90.6- \\
91.4)\end{array}$ & $\begin{array}{l}89.9(89.5- \\
90.3)\end{array}$ & $\begin{array}{l}86.6(86.1- \\
87.1)\end{array}$ & $0.707^{*}$ & $\begin{array}{l}1.06(0.91- \\
1.22)\end{array}$ & $\begin{array}{l}1.67(1.40- \\
1.99)\end{array}$ & $\begin{array}{l}1.39(1.22- \\
1.59)\end{array}$ & $\begin{array}{l}1.54(1.41- \\
1.68)\end{array}$ & $0.460^{*}$ \\
\hline Secondary & $\begin{array}{l}63.2(62.2- \\
64.1)\end{array}$ & $\begin{array}{l}84.5(83.8- \\
85.2)\end{array}$ & $\begin{array}{l}86.4(85.8- \\
86.9)\end{array}$ & $\begin{array}{l}80.3(79.6- \\
80.9)\end{array}$ & $0.009^{* *}$ & $\begin{array}{l}0.62(0.53- \\
0.72)\end{array}$ & $\begin{array}{l}1.45(1.21- \\
1.74)\end{array}$ & $\begin{array}{l}1.42(1.24- \\
1.62)\end{array}$ & $\begin{array}{l}1.21(1.12- \\
1.32)\end{array}$ & $0.113^{* *}$ \\
\hline $\begin{array}{l}\text { Higher } \\
\text { education }\end{array}$ & $\begin{array}{l}53.7(52.1- \\
55.2)\end{array}$ & $\begin{array}{l}84.5(83.5- \\
85.6)\end{array}$ & $\begin{array}{l}83.7(82.9- \\
84.5)\end{array}$ & $\begin{array}{l}77.6(76.8- \\
78.3)\end{array}$ & $0.125^{* *}$ & $\begin{array}{l}0.44(0.38- \\
0.52)\end{array}$ & $\begin{array}{l}1.43(1.18- \\
1.72)\end{array}$ & $\begin{array}{l}1.38(1.20- \\
1.59)\end{array}$ & $\begin{array}{l}1.27(1.16- \\
1.39)\end{array}$ & $0.122^{* *}$ \\
\hline Unspecified & $\begin{array}{l}56.1(45.3- \\
66.8)\end{array}$ & $\begin{array}{l}89.1(83.6- \\
94.6)\end{array}$ & $\begin{array}{l}87.5(81.5- \\
93.6)\end{array}$ & $\begin{array}{l}76.9(71.6- \\
82.2)\end{array}$ & $0.085^{* *}$ & $\begin{array}{l}0.51(0.32- \\
0.82)\end{array}$ & $\begin{array}{l}2.87(1.62- \\
5.49)\end{array}$ & $\begin{array}{l}1.46(0.84- \\
2.71)\end{array}$ & $\begin{array}{l}1.04(0.76- \\
1.42)\end{array}$ & $0.558^{* *}$ \\
\hline
\end{tabular}

$\mathrm{Cl}$ Confidence interval, OR Odds ratio adjusted for all other variables in the table, Ref Reference group

* = linear model; ${ }^{* *}=$ quadratic model

\section{Discussion}

The present study was the first to determine 15-year trends and sociodemographic correlates of meeting the new integrated 24-h movement guidelines using nationally representative time-use data. From these selfreported data, we found that only one in five Thai adults met the overall 24-h movement guidelines. However, the majority of Thai adults met the MVPA recommendation, slightly above half met the sleep recommendation, and slightly below half met the SB recommendation. Moreover, in contrast to our hypotheses, there was an increase over time in the number of Thai adults who met the $\mathrm{SB}$ recommendation, while the prevalence of meeting the MVPA and sleep recommendations peaked in 2004 and 2009 and slightly declined in 2015.

Our findings suggest that changes in movement behaviours over time among Thai adults do not align with the global trends [39, 52-54, 77]. We analysed the trends in SB based on the percentage of adults who do not engage in long periods of uninterrupted $\mathrm{SB}$, and, therefore, our findings are not directly comparable with SB trends from previous studies where the overall duration of SB was analysed. The rise in the prevalence of meeting the MVPA (from 2001 to 2004) and SB recommendations may be due to the establishment of national organizations to promote PA in Thailand in the early 2000s [47]. The reasons for a decline in MVPA since 2009 might be associated with Thailand's economic development and its transition to an upper-middle income country in 2011. This has coincided with a transition towards a more urban society [78], changes in job characteristics and the living environment, which may result in reduced opportunities to engage in MVPA in everyday life. Insufficient PA is associated with a lower likelihood of meeting the sleep recommendations [79-82], which may explain the slight decrease in the prevalence of Thai adults meeting the sleep recommendation in the same period.

In regard to the sociodemographic correlates of meeting the 24-h movement guidelines, employed adults were more likely to meet every recommendation (i.e., MVPA, MVPA for additional health benefits, SB, sleep, and overall 24-h movement guidelines) compared to adults who were unemployed. Thai adults who were married were found to be more likely to meet the MVPA, SB, sleep, and overall 24-h movement guidelines compared to those who have never been married. Thai females tended to meet the SB, sleep, and the overall guidelines more than males. However, we found that the percentage of males meeting the SB and overall guidelines increased over the 15 years. Education level seemed to play an important role in meeting the guidelines: those with no formal education were found to be the least likely to meet the MVPA, sleep, and MVPA recommendations for additional health benefits compared to other education groups. During the study period, the likelihood of meeting the overall 24-h movement guidelines was found to increase significantly for adults who had higher education.

We found that around one in five Thai adults met the overall 24-h movement guidelines (i.e., MVPA, SB, and sleep recommendations combined) in 2015. A recent study found that in the same year only $0.4 \%$ of Korean adults met a similar 'ideal' combination of movement behaviours [42]. However, a direct comparison between these findings is not possible, as there are differences in methodology used to assess movement behaviours and categorise participants. For example, the Korean study used separate questionnaires to estimate daily MVPA and $\mathrm{SB}$, and a single question for sleep duration (h), rather than a 24-h time-use dairy. The overall 24-h movement behaviour guideline in the Korean study included 
Table 4 Meeting the sedentary behaviour guideline: population prevalence and associations with sociodemographic variables

\begin{tabular}{|c|c|c|c|c|c|c|c|c|c|c|}
\hline \multirow{2}{*}{$\begin{array}{l}\text { Sociodemographic } \\
\text { variable }\end{array}$} & \multicolumn{5}{|c|}{ Percentage $(95 \% \mathrm{Cl})$} & \multicolumn{5}{|c|}{ Adjusted OR (95\% Cl) } \\
\hline & 2001 & 2004 & 2009 & 2015 & $p$-value & 2001 & 2004 & 2009 & 2015 & $\begin{array}{l}p- \\
\text { value }\end{array}$ \\
\hline Total $(n)$ & $\begin{array}{l}36.9(36.5- \\
37.4)\end{array}$ & $\begin{array}{l}39.6(39.1- \\
40.1)\end{array}$ & $\begin{array}{l}44.7(44.2- \\
45.1)\end{array}$ & $\begin{array}{l}44.6(44.1- \\
45.0)\end{array}$ & $0.002^{*}$ & & & & & \\
\hline \multicolumn{11}{|l|}{ Sex } \\
\hline Male & $\begin{array}{l}35.7(35.0- \\
36.4)\end{array}$ & $\begin{array}{l}36.4(35.7- \\
37.0)\end{array}$ & $\begin{array}{l}38.5(37.8- \\
39.1)\end{array}$ & $\begin{array}{l}41.7(41.0- \\
42.3)\end{array}$ & $\begin{array}{l}< \\
0.001^{*}\end{array}$ & Ref & & & & \\
\hline Female & $\begin{array}{l}38.2(37.5- \\
38.9)\end{array}$ & $\begin{array}{l}42.9(42.2- \\
43.6)\end{array}$ & $\begin{array}{l}50.7(50.1- \\
51.4)\end{array}$ & $\begin{array}{l}47.4(46.8- \\
48.0)\end{array}$ & $0.079^{*}$ & $\begin{array}{l}1.23(1.18- \\
1.29)\end{array}$ & $\begin{array}{l}1.47(1.40- \\
1.53)\end{array}$ & $\begin{array}{l}1.85(1.78- \\
1.93)\end{array}$ & $\begin{array}{l}1.44(1.38- \\
1.50)\end{array}$ & $0.147^{* *}$ \\
\hline \multicolumn{11}{|l|}{ Age } \\
\hline $18-29$ & $\begin{array}{l}34.1(33.3- \\
34.9)\end{array}$ & $\begin{array}{l}37.9(37.1- \\
38.7)\end{array}$ & $\begin{array}{l}41.8(41.0- \\
42.6)\end{array}$ & $\begin{array}{l}41.8(40.9- \\
42.6)\end{array}$ & $\begin{array}{l}< \\
0.001^{* *}\end{array}$ & Ref & & & & \\
\hline $30-39$ & $\begin{array}{l}39.2(38.3- \\
40.1)\end{array}$ & $\begin{array}{l}42.6(41.7- \\
43.6)\end{array}$ & $\begin{array}{l}49.8(48.9- \\
50.7)\end{array}$ & $\begin{array}{l}48.0(47.1- \\
48.9)\end{array}$ & $0.025^{*}$ & $\begin{array}{l}0.88(0.83- \\
0.94)\end{array}$ & $\begin{array}{l}0.88(0.83- \\
0.94)\end{array}$ & $\begin{array}{l}1.07(1.01- \\
1.13)\end{array}$ & $\begin{array}{l}0.95(0.90- \\
1.00)\end{array}$ & $0.632^{*}$ \\
\hline $40-49$ & $\begin{array}{l}39.3(38.3- \\
40.4)\end{array}$ & $\begin{array}{l}40.2(39.2- \\
41.3)\end{array}$ & $\begin{array}{l}44.1(43.2- \\
45.0)\end{array}$ & $\begin{array}{l}45.6(44.7- \\
46.5)\end{array}$ & $\begin{array}{l}< \\
0.001^{*}\end{array}$ & $\begin{array}{l}0.83(0.78- \\
0.89)\end{array}$ & $\begin{array}{l}0.77(0.72- \\
0.82)\end{array}$ & $\begin{array}{l}0.80(0.75- \\
0.85)\end{array}$ & $\begin{array}{l}0.80(0.75- \\
0.85)\end{array}$ & $0.952^{*}$ \\
\hline $50-59$ & $\begin{array}{l}35.9(34.6- \\
37.2)\end{array}$ & $\begin{array}{l}37.3(36.1- \\
38.5)\end{array}$ & $\begin{array}{l}42.9(41.9- \\
44.0)\end{array}$ & $\begin{array}{l}43.1(42.1- \\
44.1)\end{array}$ & $0.001^{*}$ & $\begin{array}{l}0.76(0.71- \\
0.82)\end{array}$ & $\begin{array}{l}0.70(0.65- \\
0.75)\end{array}$ & $\begin{array}{l}0.81(0.75- \\
0.86)\end{array}$ & $\begin{array}{l}0.76(0.71- \\
0.81)\end{array}$ & $0.856^{*}$ \\
\hline \multicolumn{11}{|l|}{ Household area } \\
\hline Urban & $\begin{array}{l}34.9(34.1- \\
35.7)\end{array}$ & $\begin{array}{l}38.3(37.4- \\
39.1)\end{array}$ & $\begin{array}{l}45.5(44.7- \\
46.3)\end{array}$ & $\begin{array}{l}45.2(44.6- \\
45.9)\end{array}$ & $0.002^{*}$ & Ref & & & & \\
\hline Rural & $\begin{array}{l}37.9(37.3- \\
38.5)\end{array}$ & $\begin{array}{l}40.3(39.7- \\
40.9)\end{array}$ & $\begin{array}{l}44.3(43.7- \\
44.8)\end{array}$ & $\begin{array}{l}44.0(43.4- \\
44.6)\end{array}$ & $0.005^{*}$ & $\begin{array}{l}0.96(0.91- \\
1.02)\end{array}$ & $\begin{array}{l}0.93(0.87- \\
0.98)\end{array}$ & $\begin{array}{l}0.90(0.86- \\
0.95)\end{array}$ & $\begin{array}{l}0.94(0.90- \\
0.98)\end{array}$ & $0.935^{*}$ \\
\hline \multicolumn{11}{|l|}{ Region } \\
\hline Bangkok & $\begin{array}{l}35.1(33.7- \\
36.5)\end{array}$ & $\begin{array}{l}35.6(34.3- \\
36.9)\end{array}$ & $\begin{array}{l}44.2(42.8- \\
45.6)\end{array}$ & $\begin{array}{l}46.4(45.2- \\
47.6)\end{array}$ & $\begin{array}{l}< \\
0.001^{*}\end{array}$ & Ref & & & & \\
\hline Central & $\begin{array}{l}35.1(34.1- \\
36.1)\end{array}$ & $\begin{array}{l}39.5(38.5- \\
40.5)\end{array}$ & $\begin{array}{l}45.9(44.9- \\
46.8)\end{array}$ & $\begin{array}{l}44.2(43.4- \\
45.0)\end{array}$ & $0.029^{*}$ & $\begin{array}{l}0.89(0.82- \\
0.97)\end{array}$ & $\begin{array}{l}1.11(1.02- \\
1.20)\end{array}$ & $\begin{array}{l}1.12(1.04- \\
1.21)\end{array}$ & $\begin{array}{l}0.85(0.80- \\
0.91)\end{array}$ & $0.677^{*}$ \\
\hline North & $\begin{array}{l}39.4(38.3- \\
40.6)\end{array}$ & $\begin{array}{l}46.3(45.1- \\
47.4)\end{array}$ & $\begin{array}{l}46.5(45.5- \\
47.6)\end{array}$ & $\begin{array}{l}46.8(45.7- \\
47.9)\end{array}$ & $0.137^{*}$ & $\begin{array}{l}0.98(0.90- \\
1.08)\end{array}$ & $\begin{array}{l}1.46(1.34- \\
1.59)\end{array}$ & $\begin{array}{l}1.16(1.06- \\
1.25)\end{array}$ & $\begin{array}{l}0.99(0.92- \\
1.07)\end{array}$ & $0.623^{*}$ \\
\hline North-East & $\begin{array}{l}38.2(37.4- \\
39.1)\end{array}$ & $\begin{array}{l}39.0(38.2- \\
39.9)\end{array}$ & $\begin{array}{l}43.2(42.5- \\
44.0)\end{array}$ & $\begin{array}{l}42.4(41.5- \\
43.3)\end{array}$ & $0.022^{*}$ & $\begin{array}{l}0.96(0.88- \\
1.05)\end{array}$ & $\begin{array}{l}1.04(0.96- \\
1.13)\end{array}$ & $\begin{array}{l}1.03(0.95- \\
1.12)\end{array}$ & $\begin{array}{l}0.84(0.78- \\
0.90)\end{array}$ & $0.548^{*}$ \\
\hline South & $\begin{array}{l}35.0(33.6- \\
36.4)\end{array}$ & $\begin{array}{l}35.9(34.5- \\
37.3)\end{array}$ & $\begin{array}{l}44.0(42.7- \\
45.2)\end{array}$ & $\begin{array}{l}44.8(43.6- \\
46.1)\end{array}$ & $0.001^{*}$ & $\begin{array}{l}0.85(0.77- \\
0.94)\end{array}$ & $\begin{array}{l}0.95(0.86- \\
1.05)\end{array}$ & $\begin{array}{l}1.03(0.94- \\
1.12)\end{array}$ & $\begin{array}{l}0.90(0.83- \\
0.98)\end{array}$ & $0.878^{*}$ \\
\hline \multicolumn{11}{|l|}{ Marital status } \\
\hline Never married & $\begin{array}{l}26.9(26.0- \\
27.8)\end{array}$ & $\begin{array}{l}30.8(29.9- \\
31.7)\end{array}$ & $\begin{array}{l}36.5(35.5- \\
37.4)\end{array}$ & $\begin{array}{l}37.1(36.3- \\
37.9)\end{array}$ & $0.001^{*}$ & Ref & & & & \\
\hline Married & $\begin{array}{l}40.3(39.8- \\
40.9)\end{array}$ & $\begin{array}{l}42.6(42.0- \\
43.2)\end{array}$ & $\begin{array}{l}46.7(46.2- \\
47.3)\end{array}$ & $\begin{array}{l}47.4(46.8- \\
48.0)\end{array}$ & $\begin{array}{l}< \\
0.001^{*}\end{array}$ & $\begin{array}{l}1.62(1.52- \\
1.72)\end{array}$ & $\begin{array}{l}1.52(1.43- \\
1.61)\end{array}$ & $\begin{array}{l}1.41(1.34- \\
1.48)\end{array}$ & $\begin{array}{l}1.44(1.37- \\
1.51)\end{array}$ & $0.609^{*}$ \\
\hline $\begin{array}{l}\text { Formerly } \\
\text { married }\end{array}$ & $\begin{array}{l}38.6(36.6- \\
40.7)\end{array}$ & $\begin{array}{l}40.8(38.7- \\
42.9)\end{array}$ & $\begin{array}{l}51.3(49.6- \\
53.1)\end{array}$ & $\begin{array}{l}47.8(46.1- \\
49.4)\end{array}$ & $0.070^{*}$ & $\begin{array}{l}1.52(1.36- \\
1.69)\end{array}$ & $\begin{array}{l}1.45(1.30- \\
1.61)\end{array}$ & $\begin{array}{l}1.63(1.49- \\
1.78)\end{array}$ & $\begin{array}{l}1.48(1.36- \\
1.60)\end{array}$ & $0.993^{*}$ \\
\hline \multicolumn{11}{|l|}{ Religion } \\
\hline Buddhist & $\begin{array}{l}37.0(36.5- \\
37.5)\end{array}$ & $\begin{array}{l}39.9(39.3- \\
40.4)\end{array}$ & $\begin{array}{l}44.6(44.2- \\
45.1)\end{array}$ & $\begin{array}{l}44.6(44.2- \\
45.1)\end{array}$ & $0.002^{*}$ & Ref & & & & \\
\hline Non-Buddhist & $\begin{array}{l}36.7(34.5- \\
38.9)\end{array}$ & $\begin{array}{l}35.1(32.9- \\
37.3)\end{array}$ & $\begin{array}{l}45.8(43.7- \\
47.8)\end{array}$ & $\begin{array}{l}43.9(41.8- \\
46.0)\end{array}$ & $0.066^{*}$ & $\begin{array}{l}0.99(0.89- \\
1.10)\end{array}$ & $\begin{array}{l}0.92(0.82- \\
1.03)\end{array}$ & $\begin{array}{l}1.07(0.97- \\
1.17)\end{array}$ & $\begin{array}{l}0.95(0.87- \\
1.05)\end{array}$ & $0.980^{*}$ \\
\hline \multicolumn{11}{|l|}{ Employment status } \\
\hline Employed & $\begin{array}{l}40.7(40.2- \\
41.3)\end{array}$ & $\begin{array}{l}43.2(42.6- \\
43.7)\end{array}$ & $\begin{array}{l}48.0(47.5- \\
48.5)\end{array}$ & $\begin{array}{l}48.8(48.3- \\
49.3)\end{array}$ & $\begin{array}{l}< \\
0.001^{*}\end{array}$ & Ref & & & & \\
\hline Unemployed & $\begin{array}{l}18.9(18.0- \\
20.0)\end{array}$ & $\begin{array}{l}23.0(22.0- \\
24.1)\end{array}$ & $\begin{array}{l}28.2(27.2- \\
29.2)\end{array}$ & $\begin{array}{l}26.9(26.0- \\
27.8)\end{array}$ & $\begin{array}{l}< \\
0.001^{* *}\end{array}$ & $\begin{array}{l}0.34(0.31- \\
0.36)\end{array}$ & $\begin{array}{l}0.37(0.35- \\
0.39)\end{array}$ & $\begin{array}{l}0.36(0.34- \\
0.39)\end{array}$ & $\begin{array}{l}0.36(0.34- \\
0.38)\end{array}$ & $0.819^{*}$ \\
\hline
\end{tabular}


Table 4 Meeting the sedentary behaviour guideline: population prevalence and associations with sociodemographic variables (Continued)

\begin{tabular}{|c|c|c|c|c|c|c|c|c|c|c|}
\hline \multirow{2}{*}{$\begin{array}{l}\text { Sociodemographic } \\
\text { variable }\end{array}$} & \multicolumn{5}{|c|}{ Percentage $(95 \% \mathrm{Cl})$} & \multicolumn{5}{|c|}{ Adjusted OR (95\% Cl) } \\
\hline & 2001 & 2004 & 2009 & 2015 & $p$-value & 2001 & 2004 & 2009 & 2015 & $\begin{array}{l}p- \\
\text { value }\end{array}$ \\
\hline \multicolumn{11}{|c|}{ Highest education level } \\
\hline None & $\begin{array}{l}45.1(42.3- \\
47.9)\end{array}$ & $\begin{array}{l}41.3(38.5- \\
44.1)\end{array}$ & $\begin{array}{l}43.5(41.3- \\
45.6)\end{array}$ & $\begin{array}{l}43.3(41.9- \\
44.8)\end{array}$ & $0.817^{*}$ & Ref & & & & \\
\hline Primary & $\begin{array}{l}40.1(39.5- \\
40.8)\end{array}$ & $\begin{array}{l}41.8(41.2- \\
42.5)\end{array}$ & $\begin{array}{l}44.9(44.3- \\
45.6)\end{array}$ & $\begin{array}{l}45.6(44.8- \\
46.3)\end{array}$ & $\begin{array}{l}< \\
0.001^{*}\end{array}$ & $\begin{array}{l}0.70(0.62- \\
0.79)\end{array}$ & $\begin{array}{l}0.94(0.83- \\
1.07)\end{array}$ & $\begin{array}{l}0.96(0.87- \\
1.05)\end{array}$ & $\begin{array}{l}1.08(1.01- \\
1.15)\end{array}$ & $0.215^{*}$ \\
\hline Secondary & $\begin{array}{l}31.9(31.0- \\
32.8)\end{array}$ & $\begin{array}{l}37.1(36.2- \\
38.0)\end{array}$ & $\begin{array}{l}44.7(43.8- \\
45.5)\end{array}$ & $\begin{array}{l}46.8(46.0- \\
47.6)\end{array}$ & $\begin{array}{l}< \\
0.001^{*}\end{array}$ & $\begin{array}{l}0.59(0.52- \\
0.67)\end{array}$ & $\begin{array}{l}0.88(0.78- \\
1.01)\end{array}$ & $\begin{array}{l}1.01(0.92- \\
1.11)\end{array}$ & $\begin{array}{l}1.13(1.06- \\
1.21)\end{array}$ & $0.060^{*}$ \\
\hline $\begin{array}{l}\text { Higher } \\
\text { education }\end{array}$ & $\begin{array}{l}28.8(27.4- \\
30.2)\end{array}$ & $\begin{array}{l}35.6(34.2- \\
36.9)\end{array}$ & $\begin{array}{l}44.0(42.9- \\
45.1)\end{array}$ & $\begin{array}{l}40.2(39.3- \\
41.1)\end{array}$ & $\begin{array}{l}< \\
0.001^{* *}\end{array}$ & $\begin{array}{l}0.43(0.38- \\
0.50)\end{array}$ & $\begin{array}{l}0.73(0.63- \\
0.84)\end{array}$ & $\begin{array}{l}0.96(0.86- \\
1.06)\end{array}$ & $\begin{array}{l}0.90(0.84- \\
0.97)\end{array}$ & $0.048^{* *}$ \\
\hline Unspecified & $\begin{array}{l}33.1(22.9- \\
43.3)\end{array}$ & $\begin{array}{l}34.9(26.5- \\
43.3)\end{array}$ & $\begin{array}{l}73.1(65.0- \\
81.3)\end{array}$ & $\begin{array}{l}62.3(56.2- \\
68.4)\end{array}$ & $0.083^{*}$ & $\begin{array}{l}0.58(0.35- \\
0.93)\end{array}$ & $\begin{array}{l}0.75(0.50- \\
1.11)\end{array}$ & $\begin{array}{l}3.13(2.04- \\
4.90)\end{array}$ & $\begin{array}{l}1.83(1.40- \\
2.41)\end{array}$ & $0.061^{* *}$ \\
\hline
\end{tabular}

$\mathrm{Cl}$ Confidence interval, OR Odds ratio adjusted for all other variables in the table, Ref Reference group

* = linear model; ${ }^{* *}=$ quadratic model

meeting MVPA (defined as $\geq 600$ METs minute/week) and sleep (7-9h/day) recommendations and not having high sitting time ( $\geq 9 \mathrm{~h} /$ day). Besides, the Korean study had a higher upper age limit of 64 years compared to 59 years in the current study.

The prevalence of Thai adults meeting the MVPA recommendation was very high in all survey years, even with a decrease in 2009 and 2015. National data collected using PA questionnaires, such as Global Physical Activity Questionnaire (GPAQ) and International Physical Activity Questionnaire (IPAQ), suggest similarly high prevalence rates; $77.5 \%$ in $2003,74.9 \%$ in 2009 , and $80.8 \%$ in 2015 [83]. Our results for MVPA prevalence in the Thai population are concordant with previous findings mainly from low- and middle-income countries For example, the percentage of meeting MVPA recommendation in East and Southeast Asia increased from $74.3 \%$ in 2001 to $82.7 \%$ in 2016 [77]. In some countries, such as Nepal [84], the prevalence of meeting MVPA recommendation was even higher than in Thailand. In studies that relied on data from PA questionnaires, such high prevalence rates may be a consequence of self-report bias. However, given that the questions in the Thai time-use survey did not ask specifically about PA levels, it is less likely that such bias have affected the results of our study.

The increasing MVPA in the Thai population found in the current study may be a positive result of national efforts to increase population PA. Important events for the promotion of PA in Thailand were the establishment of the Thai Health Promotion Foundation in 2001 and the Division of Physical Activity and Health, Ministry of Public Health in 2002 [47]. These two organizations along with their network of alliances aim to improve the population PA by developing policies and implementing interventions. They have supported nationwide campaigns to promote PA and the development of the first national strategic plan on the promotion of PA (20182030) [48].

The process of urbanisation may have had a significant impact on PA level of Thai adults. We found that urban residence in Thailand is associated with lower prevalence of MVPA. Evidence of such association was also found in other adult populations [42, 67, 68, 70]. With increasingly evident changes in the population structure in Thailand, particularly migration of working adults to urban areas, continuing coordinated efforts are needed to prevent a potential decline in population PA. National policies should increase opportunities that support PA specifically in urban settings, such as increasing walkability areas, improving public transportation systems, and enabling safe public use of open green spaces. It is, therefore, essential to incorporate collaborations with non-health sectors such as urban planning and transport infrastructure to create such environments. With changing nature of work, PA undertaken in occupational domain is also reduced. Future efforts are, therefore, needed to promote other types of PA particularly in transport and leisure-time domains.

Our results also indicate significant differences in the likelihood of meeting the MVPA recommendation between sexes, age groups, household areas, employment status, and education levels. Consistent with previous studies, male sex, rural setting, employment, and higher education level were significant correlates of more MVPA [42, 67, 68, 70]. However, in contrast to previous findings, older age groups in Thailand had higher MVPA than the youngest group. This finding adds to the mixed evidence of associations between younger age and higher PA $[68,85]$, and suggests that more PA promotion interventions might be needed among younger adults. This may be a result of advances in screen entertainment that supports SB and is widely used particularly among younger generations [86]. Future intervention studies may 
Table 5 Meeting the sleep guideline: population prevalence and associations with sociodemographic variables

\begin{tabular}{|c|c|c|c|c|c|c|c|c|c|c|}
\hline \multirow{2}{*}{$\begin{array}{l}\text { Sociodemographic } \\
\text { variable }\end{array}$} & \multicolumn{5}{|c|}{ Percentage $(95 \% \mathrm{Cl})$} & \multicolumn{5}{|c|}{ Adjusted OR (95\% Cl) } \\
\hline & 2001 & 2004 & 2009 & 2015 & $p$-value & 2001 & 2004 & 2009 & 2015 & $\begin{array}{l}p^{-} \\
\text {value }\end{array}$ \\
\hline Total $(n)$ & $\begin{array}{l}53.7(53.2- \\
54.2)\end{array}$ & $\begin{array}{l}56.1(55.6- \\
56.6)\end{array}$ & $\begin{array}{l}57.8(57.4- \\
58.3)\end{array}$ & $\begin{array}{l}56.4(56.0- \\
56.9)\end{array}$ & $\begin{array}{l}< \\
0.001^{* *}\end{array}$ & & & & & \\
\hline \multicolumn{11}{|l|}{ Sex } \\
\hline Male & $\begin{array}{l}51.7(51.0- \\
52.4)\end{array}$ & $\begin{array}{l}54.5(53.7- \\
55.2)\end{array}$ & $\begin{array}{l}55.6(55.0- \\
56.3)\end{array}$ & $\begin{array}{l}53.7(53.0- \\
54.3)\end{array}$ & $0.494^{*}$ & Ref & & & & \\
\hline Female & $\begin{array}{l}55.7(55.0- \\
56.4)\end{array}$ & $\begin{array}{l}57.8(57.1- \\
58.5)\end{array}$ & $\begin{array}{l}60.0(59.4- \\
60.6)\end{array}$ & $\begin{array}{l}59.0(58.4- \\
59.6)\end{array}$ & $0.085^{*}$ & $\begin{array}{l}1.28(1.22- \\
1.33)\end{array}$ & $\begin{array}{l}1.25(1.20- \\
1.30)\end{array}$ & $\begin{array}{l}1.32(1.27- \\
1.37)\end{array}$ & $\begin{array}{l}1.35(1.30- \\
1.40)\end{array}$ & $0.715^{*}$ \\
\hline \multicolumn{11}{|l|}{ Age } \\
\hline $18-29$ & $\begin{array}{l}53.8(52.9- \\
54.6)\end{array}$ & $\begin{array}{l}56.2(55.4- \\
57.1)\end{array}$ & $\begin{array}{l}58.0(57.2- \\
58.8)\end{array}$ & $\begin{array}{l}54.6(53.8- \\
55.5)\end{array}$ & $0.798^{*}$ & Ref & & & & \\
\hline $30-39$ & $\begin{array}{l}56.4(55.5- \\
57.4)\end{array}$ & $\begin{array}{l}56.9(55.9- \\
57.9)\end{array}$ & $\begin{array}{l}59.6(58.8- \\
60.5)\end{array}$ & $\begin{array}{l}58.3(57.4- \\
59.2)\end{array}$ & $0.181^{*}$ & $\begin{array}{l}1.10(1.03- \\
1.16)\end{array}$ & $\begin{array}{l}0.94(0.88- \\
0.99)\end{array}$ & $\begin{array}{l}0.99(0.94- \\
1.05)\end{array}$ & $\begin{array}{l}1.02(0.96- \\
1.07)\end{array}$ & $0.900^{*}$ \\
\hline $40-49$ & $\begin{array}{l}53.9(52.9- \\
55.0)\end{array}$ & $\begin{array}{l}56.6(55.6- \\
57.7)\end{array}$ & $\begin{array}{l}57.6(56.7- \\
58.5)\end{array}$ & $\begin{array}{l}58.4(57.5- \\
59.3)\end{array}$ & $0.002^{*}$ & $\begin{array}{l}0.99(0.94- \\
1.06)\end{array}$ & $\begin{array}{l}0.95(0.89- \\
1.01)\end{array}$ & $\begin{array}{l}0.94(0.89- \\
0.99)\end{array}$ & $\begin{array}{l}1.07(1.01- \\
1.13)\end{array}$ & $0.744^{*}$ \\
\hline $50-59$ & $\begin{array}{l}48.0(46.7- \\
49.3)\end{array}$ & $\begin{array}{l}53.9(52.6- \\
55.1)\end{array}$ & $\begin{array}{l}55.5(54.4- \\
56.5)\end{array}$ & $\begin{array}{l}54.2(53.2- \\
55.2)\end{array}$ & $0.182^{*}$ & $\begin{array}{l}0.83(0.77- \\
0.89)\end{array}$ & $\begin{array}{l}0.9(0.84- \\
0.97)\end{array}$ & $\begin{array}{l}0.93(0.88- \\
0.99)\end{array}$ & $\begin{array}{l}0.96(0.91- \\
1.03)\end{array}$ & $0.563^{*}$ \\
\hline \multicolumn{11}{|l|}{ Household area } \\
\hline Urban & $\begin{array}{l}52.1(51.2- \\
53.0)\end{array}$ & $\begin{array}{l}57.0(56.1- \\
57.8)\end{array}$ & $\begin{array}{l}58.0(57.2- \\
58.8)\end{array}$ & $\begin{array}{l}58.2(57.5- \\
58.9)\end{array}$ & $0.065^{*}$ & Ref & & & & \\
\hline Rural & $\begin{array}{l}54.5(53.9- \\
55.1)\end{array}$ & $\begin{array}{l}55.7(55.1- \\
56.3)\end{array}$ & $\begin{array}{l}57.8(57.2- \\
58.3)\end{array}$ & $\begin{array}{l}54.8(54.2- \\
55.4)\end{array}$ & $0.816^{*}$ & $\begin{array}{l}1.01(0.96- \\
1.07)\end{array}$ & $\begin{array}{l}0.97(0.92- \\
1.02)\end{array}$ & $\begin{array}{l}0.97(0.93- \\
1.02)\end{array}$ & $\begin{array}{l}0.91(0.87- \\
0.95)\end{array}$ & $0.647^{*}$ \\
\hline \multicolumn{11}{|l|}{ Region } \\
\hline Bangkok & $\begin{array}{l}48.3(46.9- \\
49.8)\end{array}$ & $\begin{array}{l}57.5(56.1- \\
58.8)\end{array}$ & $\begin{array}{l}55.3(53.9- \\
56.7)\end{array}$ & $\begin{array}{l}58.7(57.5- \\
59.8)\end{array}$ & $0.123^{*}$ & Ref & & & & \\
\hline Central & $\begin{array}{l}54.9(53.9- \\
56.0)\end{array}$ & $\begin{array}{l}55.6(54.5- \\
56.6)\end{array}$ & $\begin{array}{l}59.4(58.5- \\
60.3)\end{array}$ & $\begin{array}{l}57.4(56.6- \\
58.2)\end{array}$ & $0.212^{*}$ & $\begin{array}{l}1.33(1.23- \\
1.44)\end{array}$ & $\begin{array}{l}0.94(0.87- \\
1.01)\end{array}$ & $\begin{array}{l}1.27(1.17- \\
1.37)\end{array}$ & $\begin{array}{l}1.03(0.96- \\
1.09)\end{array}$ & $0.633^{*}$ \\
\hline North & $\begin{array}{l}57.0(55.9- \\
58.2)\end{array}$ & $\begin{array}{l}56.0(54.8- \\
57.1)\end{array}$ & $\begin{array}{l}56.8(55.7- \\
57.9)\end{array}$ & $\begin{array}{l}54.8(53.7- \\
55.9)\end{array}$ & $0.103^{*}$ & $\begin{array}{l}1.46(1.34- \\
1.59)\end{array}$ & $\begin{array}{l}0.98(0.90- \\
1.07)\end{array}$ & $\begin{array}{l}1.16(1.07- \\
1.26)\end{array}$ & $\begin{array}{l}0.97(0.90- \\
1.04)\end{array}$ & $0.285^{*}$ \\
\hline North-East & $\begin{array}{l}55.5(54.6- \\
56.3)\end{array}$ & $\begin{array}{l}58.0(57.1- \\
58.9)\end{array}$ & $\begin{array}{l}60.3(59.5- \\
61.1)\end{array}$ & $\begin{array}{l}57.7(56.8- \\
58.6)\end{array}$ & $0.434^{*}$ & $\begin{array}{l}1.36(1.25- \\
1.47)\end{array}$ & $\begin{array}{l}1.05(0.97- \\
1.14)\end{array}$ & $\begin{array}{l}1.38(1.27- \\
1.49)\end{array}$ & $\begin{array}{l}1.12(1.04- \\
1.20)\end{array}$ & $0.741^{*}$ \\
\hline South & $\begin{array}{l}46.7(45.2- \\
48.1)\end{array}$ & $\begin{array}{l}51.0(49.5- \\
52.4)\end{array}$ & $\begin{array}{l}52.5(51.2- \\
53.7)\end{array}$ & $\begin{array}{l}51.1(49.8- \\
52.3)\end{array}$ & $0.220^{*}$ & $\begin{array}{l}0.93(0.85- \\
1.02)\end{array}$ & $\begin{array}{l}0.78(0.71- \\
0.86)\end{array}$ & $\begin{array}{l}0.95(0.87- \\
1.04)\end{array}$ & $\begin{array}{l}0.81(0.75- \\
0.87)\end{array}$ & $0.800^{*}$ \\
\hline \multicolumn{11}{|l|}{ Marital status } \\
\hline Never married & $\begin{array}{l}53.2(52.2- \\
54.2)\end{array}$ & $\begin{array}{l}53.0(52.0- \\
54.0)\end{array}$ & $\begin{array}{l}56.2(55.2- \\
57.1)\end{array}$ & $\begin{array}{l}54.7(53.9- \\
55.6)\end{array}$ & $0.229^{*}$ & Ref & & & & \\
\hline Married & $\begin{array}{l}54.2(53.6- \\
54.8)\end{array}$ & $\begin{array}{l}57.6(57.0- \\
58.2)\end{array}$ & $\begin{array}{l}58.8(58.2- \\
59.3)\end{array}$ & $\begin{array}{l}57.5(57.0- \\
58.1)\end{array}$ & $0.250^{*}$ & $\begin{array}{l}0.98(0.93- \\
1.04)\end{array}$ & $\begin{array}{l}1.20(1.13- \\
1.27)\end{array}$ & $\begin{array}{l}1.10(1.04- \\
1.16)\end{array}$ & $\begin{array}{l}1.10(1.05- \\
1.15)\end{array}$ & $0.814^{*}$ \\
\hline $\begin{array}{l}\text { Formerly } \\
\text { married }\end{array}$ & $\begin{array}{l}49.9(47.8- \\
52.0)\end{array}$ & $\begin{array}{l}52.1(50.0- \\
54.2)\end{array}$ & $\begin{array}{l}54.0(52.3- \\
55.8)\end{array}$ & $\begin{array}{l}53.2(51.6- \\
54.8)\end{array}$ & $0.101^{*}$ & $\begin{array}{l}0.85(0.77- \\
0.94)\end{array}$ & $\begin{array}{l}1.00(0.90- \\
1.11)\end{array}$ & $\begin{array}{l}0.91(0.84- \\
0.99)\end{array}$ & $\begin{array}{l}0.93(0.85- \\
1.00)\end{array}$ & $0.924^{*}$ \\
\hline \multicolumn{11}{|l|}{ Religion } \\
\hline Buddhist & $\begin{array}{l}53.9(53.3- \\
54.4)\end{array}$ & $\begin{array}{l}56.4(55.9- \\
56.9)\end{array}$ & $\begin{array}{l}58.0(57.6- \\
58.5)\end{array}$ & $\begin{array}{l}56.6(56.1- \\
57.0)\end{array}$ & $\begin{array}{l}< \\
0.001^{* *}\end{array}$ & Ref & & & & \\
\hline Non-Buddhist & $\begin{array}{l}50.7(48.4- \\
53.0)\end{array}$ & $\begin{array}{l}51.2(48.9- \\
53.5)\end{array}$ & $\begin{array}{l}54.2(52.1- \\
56.2)\end{array}$ & $\begin{array}{l}53.3(51.2- \\
55.4)\end{array}$ & $0.070^{*}$ & $\begin{array}{l}1.07(0.97- \\
1.19)\end{array}$ & $\begin{array}{l}1.00(0.90- \\
1.11)\end{array}$ & $\begin{array}{l}1.04(0.95- \\
1.14)\end{array}$ & $\begin{array}{l}1.08(0.98- \\
1.18)\end{array}$ & $0.931^{*}$ \\
\hline \multicolumn{11}{|l|}{ Employment status } \\
\hline Employed & $\begin{array}{l}55.1(54.6- \\
55.7)\end{array}$ & $\begin{array}{l}58.2(57.6- \\
58.7)\end{array}$ & $\begin{array}{l}60.1(59.6- \\
60.6)\end{array}$ & $\begin{array}{l}58.8(58.4- \\
59.3)\end{array}$ & $0.149^{*}$ & Ref & & & & \\
\hline Unemployed & $\begin{array}{l}46.9(45.7- \\
48.2)\end{array}$ & $\begin{array}{l}46.6(45.4- \\
47.8)\end{array}$ & $\begin{array}{l}47.0(45.9- \\
48.1)\end{array}$ & $\begin{array}{l}46.1(45.1- \\
47.2)\end{array}$ & $0.353^{*}$ & $\begin{array}{l}0.69(0.65- \\
0.73)\end{array}$ & $\begin{array}{l}0.60(0.56- \\
0.63)\end{array}$ & $\begin{array}{l}0.54(0.51- \\
0.57)\end{array}$ & $\begin{array}{l}0.55(0.53- \\
0.58)\end{array}$ & $0.351^{*}$ \\
\hline
\end{tabular}


Table 5 Meeting the sleep guideline: population prevalence and associations with sociodemographic variables (Continued)

\begin{tabular}{|c|c|c|c|c|c|c|c|c|c|c|}
\hline \multirow{2}{*}{$\begin{array}{l}\text { Sociodemographic } \\
\text { variable }\end{array}$} & \multicolumn{5}{|c|}{ Percentage (95\% Cl) } & \multicolumn{5}{|c|}{ Adjusted OR (95\% Cl) } \\
\hline & 2001 & 2004 & 2009 & 2015 & $p$-value & 2001 & 2004 & 2009 & 2015 & $\begin{array}{l}p^{-} \\
\text {value }\end{array}$ \\
\hline \multicolumn{11}{|c|}{ Highest education level } \\
\hline None & $\begin{array}{l}45.0(42.2- \\
47.8)\end{array}$ & $\begin{array}{l}44.7(41.9- \\
47.5)\end{array}$ & $\begin{array}{l}50.2(48.0- \\
52.3)\end{array}$ & $\begin{array}{l}52.3(50.9- \\
53.8)\end{array}$ & $\begin{array}{l}< \\
0.001^{*}\end{array}$ & Ref & & & & \\
\hline Primary & $\begin{array}{l}53.9(53.3- \\
54.5)\end{array}$ & $\begin{array}{l}55.3(54.6- \\
56.0)\end{array}$ & $\begin{array}{l}55.8(55.1- \\
56.4)\end{array}$ & $\begin{array}{l}54.6(53.8- \\
55.3)\end{array}$ & $0.695^{*}$ & $\begin{array}{l}1.41(1.25- \\
1.59)\end{array}$ & $\begin{array}{l}1.40(1.24- \\
1.58)\end{array}$ & $\begin{array}{l}1.14(1.04- \\
1.25)\end{array}$ & $\begin{array}{l}1.05(0.98- \\
1.13)\end{array}$ & $0.261^{*}$ \\
\hline Secondary & $\begin{array}{l}54.0(53.0- \\
55.0)\end{array}$ & $\begin{array}{l}57.1(56.2- \\
58.1)\end{array}$ & $\begin{array}{l}59.4(58.5- \\
60.2)\end{array}$ & $\begin{array}{l}56.6(55.8- \\
57.4)\end{array}$ & $\begin{array}{l}< \\
0.001^{* *}\end{array}$ & $\begin{array}{l}1.53(1.35- \\
1.73)\end{array}$ & $\begin{array}{l}1.68(1.48- \\
1.90)\end{array}$ & $\begin{array}{l}1.42(1.29- \\
1.56)\end{array}$ & $\begin{array}{l}1.19(1.11- \\
1.27)\end{array}$ & $0.275^{*}$ \\
\hline $\begin{array}{l}\text { Higher } \\
\text { education }\end{array}$ & $\begin{array}{l}54.5(52.9- \\
56.0)\end{array}$ & $\begin{array}{l}60.1(58.7- \\
61.5)\end{array}$ & $\begin{array}{l}63.2(62.1- \\
64.2)\end{array}$ & $\begin{array}{l}60.8(59.9- \\
61.8)\end{array}$ & $\begin{array}{l}< \\
0.001^{* *}\end{array}$ & $\begin{array}{l}1.48(1.29- \\
1.69)\end{array}$ & $\begin{array}{l}1.73(1.51- \\
1.98)\end{array}$ & $\begin{array}{l}1.67(1.51- \\
1.85)\end{array}$ & $\begin{array}{l}1.43(1.33- \\
1.54)\end{array}$ & $0.740^{*}$ \\
\hline Unspecified & $\begin{array}{l}58.2(47.5- \\
68.9)\end{array}$ & $\begin{array}{l}63.1(54.5- \\
71.6)\end{array}$ & $\begin{array}{l}53.0(43.8- \\
62.2)\end{array}$ & $\begin{array}{l}50.9(44.6- \\
57.2)\end{array}$ & $0.045^{*}$ & $\begin{array}{l}1.92(1.22- \\
3.06)\end{array}$ & $\begin{array}{l}2.02(1.38- \\
3.00)\end{array}$ & $\begin{array}{l}1.07(0.73- \\
1.58)\end{array}$ & $\begin{array}{l}0.86(0.66- \\
1.11)\end{array}$ & $0.091^{*}$ \\
\hline
\end{tabular}

$\mathrm{Cl}$ Confidence interval, OR Odds ratio adjusted for all other variables in the table, Ref Reference group

* = linear model; ${ }^{*}=$ quadratic model

consider exploring possible uses of emerging media and new technologies, which are increasing in popularity among young adults, to promote PA [87].

We found that more than a half of Thai adults do not interrupt their sedentary activities at least every $2 \mathrm{~h}$. It is encouraging that the number of Thai adults who meet the SB recommendation is increasing. This may be due to a rapid development of the body of evidence on detrimental effects of SB that occurred in the first half of the previous decade. The odds of meeting the SB recommendation were significantly higher among Thai females when compared with their male counterparts. This result supports the finding of a recent systematic review on SB correlates in Thailand that male adults engage more in SB than females [85]. This result is, however, contradictory to the finding of a previous review that included studies from other countries [88]. The reason for this difference may be that most previous evidence on this association came from high-income countries. Moreover, in accordance with existing literature, our study confirms that older age, being single, and being unemployed have positive associations with SB [88].

To our knowledge, this study is the first to examine the trends in meeting the sleep recommendation in Thai adults. Only about a half of Thai adults met the sleep recommendation. The prevalence of Thai adults meeting the sleep recommendation increased over the study period (except for a slight decrease in 2015). In comparison, no trends were observed in several other countries [52-54, 89], while a declining trend was found in the American adult population from 2004 to 2012 [90]. In 2015 , the prevalence of meeting sleep recommendation among Thai adults was similar to that for Korean adults [42]. This study explored sleep duration only, while sleep quality has not been examined. As there is limited information about trends in sleep among Thai adults, more studies are needed in this area, particularly including measures of different aspects of sleep quality.

\section{Strengths and limitations}

There are several strengths of the present study. First, this is the first study to examine the prevalence of meeting the Thai 24-h movement recommendations. Second, a large sample size ensured adequate statistical power and sufficient precision in estimating population effect size. Third, this study showcases how to employ ICATUS-based data in PA and SB research. Last, we used time-use survey data which have been shown to provide reliable and valid estimates in large-scale studies [23].

This study had limitations. Firstly, ICATUS does not include a detailed breakdown of occupation- and travelrelated activities according to their intensity level and posture. A more detailed assessment of these activities would allow for a more precise quantification of PA and SB levels. Using device-based measures of PA and SB might improve the accuracy of prevalence estimates. However, due to time and cost limitations, device-based measurements may be challenging to use for the assessment of PA and SB in large-scale studies, particularly in low- and middle-income countries [36, 91]. Also, the activities were reported in 10-min reference periods for the last three observations. This may have led to an underestimation of the prevalence of meeting the SB guideline because no data was available on possible short interruptions of SB within these reference periods. Secondly, the Thai national time-use surveys were based on a one-day diary. Given that movement behaviours may vary across days of measurement, a longer measurement period would likely provide more reliable individual estimates. Thirdly, the time-use surveys used to determine population-level estimates in this study were collected prior to the release of the Thai 24-h guidelines. The movement behaviours of the Thai population may have changed now that the guidelines have been published. This should be examined in future surveys. Finally, in this study we did not analyse domain-specific data on 
PA and SB (e.g. at work, in household, in transport, and in leisure-time). Such an additional analysis would provide even deeper understanding of patterns of these behaviours in the Thai population, but it was beyond the scope of this paper.

\section{Recommendations for future research}

Prevalence and correlates of meeting the 24-h guidelines should be comprehensively explored in different countries and world regions, because they may vary across different social, environmental, cultural, and political contexts. Besides the assessment of time use, most timeuse surveys include only a limited number of questions on sociodemographic characteristics of participants. Future studies in this area would benefit from investigating a wider range of correlates, beyond only individual-level sociodemographic factors. Future research should also consider: 1) using more detailed questions on the time spent in occupation- and travel-related activities; 2) assessing time use over multiple days; and 3) analysing correlates of domain-specific PA and SB.

\section{Conclusions}

Based on self-reported time-use data, we found that, despite promising trends in the prevalence of meeting $\mathrm{PA}, \mathrm{SB}$, and sleep recommendations, a large majority of Thai adults do not meet the overall 24-h movement guidelines. Further actions are needed to promote more MVPA, less SB, and adequate sleep in Thai adults, particularly among males, those living in urban areas, inhabitants of Bangkok and South Thailand, unemployed, and those with low education level. To be able to assess the effectiveness of such actions at the national level, it is critical to maintain regular nationwide surveillance and monitoring systems. Future studies should explore in more detail sleep quality and patterns of PA and SB in the Thai population. Moreover, since the 24-h guidelines were only recently issued in Thailand, it is important to disseminate them, to ensure they reach as many people as possible. Our findings indicate that, nationwide public health actions are needed to promote more $\mathrm{PA}$, less $\mathrm{SB}$, and adequate sleep among Thai adults, to increase the prevalence of meeting 24-h guidelines.

\section{Supplementary information}

Supplementary information accompanies this paper at https://doi.org/10. 1186/s12966-020-01011-9.

Additional file 1. Meeting the MVPA recommendation for additional health benefits. The prevalence and association table of MVPA for additional health benefits.

\section{Abbreviations}

AIC: Akaike Information Criterion; ATUS: American Time Use Survey; BMI: Body mass index; GPAQ: Global Physical Activity Questionnaire;
ICATUS: International Classification of Activities for Time-Use Statistics: IPAQ: International Physical Activity Questionnaire; ISCO: International Standard Classification of Occupations; LPA: Light physical activity; METs: Metabolic equivalents; MVPA: Moderate-to-vigorous physical activity; NSO: National Statistical Office; PA: Physical activity; REML: Restricted Maximum Likelihood Estimation; SB: Sedentary behaviour; GSS-TU: Statistics Canada's General Social Survey - Time Use; WHO: World Health Organization

\section{Acknowledgments}

We acknowledge the National Statistical Office (NSO) for providing raw data of the 2001, 2004, 2009, and 2015 Thai National Time-Use Survey. This paper is a part of a PhD project of the first author, $\mathrm{NL}$, under supervision of $\mathrm{MC}$, $\mathrm{SJHB}$, and ZP (principal supervisor).

\section{Authors' contributions}

$\mathrm{NL}$ and ZP conceived the idea for the study. NL, ZP, DD, MC, and SJHB conceptualised the study. NL, ZP and DD conceptualised the data analysis approach. NL conducted the harmonisation of ICATUS activities and analysed the datasets. NL drafted the manuscript. ZP, DD, MC, and SJHB contributed to writing the manuscript. All authors read and approved the final draft and all revisions of the manuscript.

\section{Funding}

DD is supported by the National Health and Medical Research Council (NHMRC) [Early Career Fellowship 1162166] and the National Heart Foundation of Australia [102084].

\section{Availability of data and materials}

The datasets analysed in the current study are available from the NSO, but restrictions apply to the availability of these data. Given that a permission from NSO is needed to access and use the data, they cannot be made publicly available alongside this paper. The data are, however, available from the first author upon a reasonable request and with written permission from the NSO.

\section{Ethics approval and consent to participate}

The Thai National Time-Use Surveys were conducted by the National Statistical Office (NSO), a state agency responsible for collecting and analysing statistical data for the national development. The NSO is enacted by the Official Information Act, B.E. 2540 (1997), and it requires from all participants to provide informed consent prior to participating in the surveys. Participants' anonymity and confidentiality of their data were ensured and protected by the legislation. This study had NSO permissions for the use of 2001, 2004, 2009, and 2015 Thai Time-Use Surveys.

Consent for publication

Not applicable.

\section{Competing interests}

The authors declare no competing interests.

\section{Author details}

${ }^{1}$ Institute for Health and Sport, Victoria University, PO Box 14428, Melbourne, VIC 8001, Australia. ${ }^{2}$ Institute for Population and Social Research, Mahidol University, Phutthamonthon Sai 4 Road, Salaya, Phutthamonthon, Nakhon Pathom 73170, Thailand. ${ }^{3}$ Allied Health and Human Performance, Alliance for Research in Exercise, Nutrition and Activity, University of South Australia, Frome Road, Adelaide, South Australia 5001, Australia. ${ }^{4}$ Mitchell Institute for Education and Health Policy, Victoria University, PO Box 14428, Melbourne, VIC 8001, Australia. ${ }^{5}$ Centre for Health Research, University of Southern Queensland, Education City, 37 Sinnathamby Boulevard, Springfield Central, Queensland 4300, Australia.

Received: 28 February 2020 Accepted: 10 August 2020 Published online: 24 August 2020

\section{References}

1. Chastin SF, Palarea-Albaladejo J. Concise guide to compositional data analysis for physical activity, sedentary behavior, and sleep research: supplementary material S2, in Chastin SFM, Palarea-Albaladejo J, Dontje ML, Skelton DA. "Combined effects of time spent in physical activity, sedentary 
behaviors and sleep on obesity and cardio-metabolic health markers: a novel compositional data analysis approach". PLoS One. 2015;10(10):e0139984.

2. Dumuid D, Stanford TE, Martin-Fernández J-A, Pedišić Ž, Maher CA, Lewis LK, et al. Compositional data analysis for physical activity, sedentary time and sleep research. Stat Methods Med Res. 2018;27(12):3726-38.

3. Pedišić Ž, Dumuid D, Olds TS. Integrating sleep, sedentary behaviour, and physical activity research in the emerging field of time-use epidemiology: definitions, concepts, statistical methods, theoretical framework, and future directions. Kinesiology. 2017;49(2):252-69.

4. Pedišić Ž. Measurement issues and poor adjustments for physical activity and sleep undermine sedentary behaviour research - the focus should shift to the balance between sleep, sedentary behaviour, standing and activity. Kinesiology. 2014;46(1):135-46.

5. Carson V, Tremblay MS, Chaput J-P, Chastin SF. Associations between sleep duration, sedentary time, physical activity, and health indicators among Canadian children and youth using compositional analyses. Appl Physiol Nutr Metab. 2016;41(6):S294-302.

6. Chastin SF, Palarea-Albaladejo J, Dontje ML, Skelton DA. Combined effects of time spent in physical activity, sedentary behaviors and sleep on obesity and cardio-metabolic health markers: a novel compositional data analysis approach. PLoS One. 2015;10(10):e0139984.

7. McGregor D, Palarea-Albaladejo J, Dall P, Stamatakis E, Chastin S. Differences in physical activity time-use composition associated with cardiometabolic risks. Prev Med Rep. 2019;13:23-9.

8. McGregor D, Carson V, Palarea-Albaladejo J, Dall P, Tremblay M, Chastin S. Compositional analysis of the associations between 24-h movement behaviours and health indicators among adults and older adults from the Canadian health measure survey. Int J Environ Res Public Health. 2018;15(8):1779.

9. Dumuid D, Stanford TE, Pedišić Ž, Maher C, Lewis LK, Martín-Fernández J-A, et al. Adiposity and the isotemporal substitution of physical activity, sedentary time and sleep among school-aged children: a compositional data analysis approach. BMC Public Health. 2018;18(1):311.

10. Gupta N, Korshøj M, Dumuid D, Coenen P, Allesøe K, Holtermann A. Daily domain-specific time-use composition of physical behaviors and blood pressure. Int J Behav Nutr Phys Act. 2019;16(1):4.

11. Okely AD, Ghersi D, Hesketh KD, Santos R, Loughran SP, Cliff DP, et al. A collaborative approach to adopting/adapting guidelines-the Australian 24-hour movement guidelines for the early years (birth to 5 years): an integration of physical activity, sedentary behavior, and sleep. BMC Public Health. 2017;17(5):869.

12. New Zealand Ministry of Health. Sit less, move more, sleep well: physical activity guidelines for children and young people. Wellington: Ministry of Health; 2017.

13. Khamput T, Phuangkrampun M, Sangsumritpol W, Thongbo T, Sianglee S, Kaeyai T. Thailand recommendations on physical activity, non-sedentary lifestyles, and sleeping. 1st ed. Nonthaburi: Division of Physical Activity and Health, Ministry of Public Health; 2017.

14. Draper CE, Tomaz SA, Biersteker L, Cook CJ, Couper J, de Milander M, et al, The south African 24-hour movement guidelines for birth to 5 years: an integration of physical activity, sitting behavior, screen time, and sleep. $J$ Phys Act Health. 2020;17(1):109-19.

15. UKK Institute for Health Promotion Research. Aikuisten liikkumisen suositus [Movement recommendations for adults]. Tampere: UKK Institute for Health Promotion Research; 2019. Available from: https://www.ukkinstituutti.fi/ liikkumisensuositus/aikuisten-liikkumisen-suositus.

16. Tremblay MS, Carson V, Chaput J-P, Connor Gorber S, Dinh T, Duggan M, et al. Canadian 24-hour movement guidelines for children and youth: an integration of physical activity, sedentary behaviour, and sleep. Appl Physiol Nutr Metab. 2016:41(6):S311-S27.

17. Reilly JJ, Hughes AR, Janssen $X$, Hesketh KR, Livingstone S, Hill C, et al. GRADE-ADOLOPMENT process to develop 24-hour movement behavior recommendations and physical activity guidelines for the under $5 \mathrm{~s}$ in the United Kingdom, 2019. J Phys Act Health. 2020;17(1):101-8.

18. Jurakic D, Pedisic Z. Croatian 24-hour guidelines for physical activity, sedentary behaviour, and sleep: a proposal based on a systematic review of literature. Medicus. 2019;28(2):143-53.

19. World Health Organization. Guidelines on physical activity, sedentary behaviour and sleep for children under 5 years of age. Geneva: World Health Organization; 2019. Available from: http://www.who.int/iris/handle/10665/311664.

20. United Nations Statistics Division. Gender statistics: Department of Economic and Social Affairs, United Nations; 2018. Available from: https:// unstats.un.org/unsd/gender/timeuse/.
21. Bauman A, Bittman M, Gershuny J. A short history of time use research; implications for public health. BMC Public Health. 2019;19(2):607.

22. Tremblay MS. Introducing 24-hour movement guidelines for the early years: a new paradigm gaining momentum. J Phys Act Health. 2019;17:92-5.

23. van der Ploeg HP, Merom D, Chau JY, Bittman M, Trost SG, Bauman AE. Advances in population surveillance for physical activity and sedentary behavior: reliability and validity of time use surveys. Am J Epidemiol. 2010; 172(10):1199-206.

24. Matthews CE, Keadle SK, Sampson J, Lyden K, Bowles HR, Moore SC, et al. Validation of a previous-day recall measure of active and sedentary behaviors. Med Sci Sports Exerc. 2013:45(8):1629-38.

25. Kelly P, Thomas E, Doherty A, Harms T, Burke Ó, Gershuny J, et al. Developing a method to test the validity of 24 hour time use diaries using wearable cameras: a feasibility pilot. PLoS One. 2015;10(12):e0142198.

26. Ibáñez V, Silva J, Cauli O. A survey on sleep assessment methods. PeerJ. 2018;6:e4849.

27. Tudor-Locke C, Bittman M, Merom D, Bauman A. Patterns of walking for transport and exercise: a novel application of time use data. Int J Behav Nutr Phys Act. 2005;2(1):5.

28. Tudor-Locke C, van der Ploeg HP, Bowles HR, Bittman M, Fisher K, Merom D, et al. Walking behaviours from the 1965-2003 American heritage time use study (AHTUS). Int J Behav Nutr Phys Act. 2007;4(1):45.

29. Espinel PT, Chau JY, van der Ploeg HP, Merom D. Older adults' time in sedentary, light and moderate intensity activities and correlates: application of Australian time use survey. J Sci Med Sport. 2015;18(2):161-6.

30. van Tienoven TP, Deyaert J, Harms T, Weenas D, Minnen J, Glorieux I. Active work, passive leisure? Associations between occupational and nonoccupational physical activity on weekdays. Soc Sci Res. 2018;76:1-11.

31. Millward H, Spinney J. "Active living" related to the rural-urban continuum: a time-use perspective. J Rural Health. 2011;27(2):141-50.

32. Millward H, Spinney J, Scott D. Active-transport walking behavior: destinations, durations, distances. J Transp Geogr. 2013;28:101-10.

33. Millward H, Spinney JE, Scott D. Durations and domains of daily aerobic activity: evidence from the 2010 Canadian time-use survey. J Phys Act Health. 2014;11(5):895-902.

34. Spinney JE. Aerobic activity preferences among older Canadians: a time use perspective. Can J Aging/La Revue canadienne du vieillissement. 2013;32(4): $443-51$.

35. Spinney JE, Scott DM, Newbold KB. Transport mobility benefits and quality of life: a time-use perspective of elderly Canadians. Transp Policy. 2009;16(1):1-11.

36. Harms T, Berrigan D, Gershuny J. Daily metabolic expenditures: estimates from US, UK and polish time-use data. BMC Public Health. 2019;19(2):453.

37. Loyen A, Chau JY, Jelsma JG, van Nassau F, van der Ploeg HP. Prevalence and correlates of domain-specific sedentary time of adults in the Netherlands: findings from the 2006 Dutch time use survey. BMC Public Health. 2019;19(2):538.

38. Chau JY, Merom D, Grunseit A, Rissel C, Bauman AE, van der Ploeg HP. Temporal trends in non-occupational sedentary behaviours from Australian time use surveys 1992, 1997 and 2006. Int J Behav Nutr Phys Act. 2012;9(1):76.

39. Ng SW, Popkin BM. Time use and physical activity: a shift away from movement across the globe. Obes Rev. 2012;13(8):659-80.

40. Spinney JE, Millward H. Active living among older Canadians: a time-use perspective over 3 decades. J Aging Phys Act. 2014;22(1):103-13.

41. Spinney JE, Millward H, Scott DM. Measuring active living in Canada: a timeuse perspective. Soc Sci Res. 2011;40(2):685-94.

42. Lee E-Y, Carson V, Jeon JY, Spence JC, Tremblay MS. Levels and correlates of 24-hour movement behaviors among South Koreans: results from the Korea National Health and Nutrition Examination Surveys, 2014 and 2015. J Sport Health Sci. 2019:8(4):376-85.

43. Lee E-Y, Spence JC, Tremblay MS, Carson V. Meeting 24-hour movement guidelines for children and youth and associations with psychological wellbeing among south Korean adolescents. Ment Health Phys Act. 2018;14:66-73.

44. Carson V, Chaput J-P, Janssen I, Tremblay MS. Health associations with meeting new 24-hour movement guidelines for Canadian children and youth. Prev Med. 2017;95:7-13.

45. Sampasa-Kanyinga $\mathrm{H}$, Standage M, Tremblay MS, Katzmarzyk P, Hu G, Kuriyan R, et al. Associations between meeting combinations of 24-h movement guidelines and health-related quality of life in children from 12 countries. Public Health. 2017;153:16-24.

46. Health Insurance System Research Office. Thailand's universal coverage scheme: achievements and challenges, an independent assessment of the 
first 10 years (2001-2010): Center for Global Development; 2012. Available from: http://millionssaved.cgdev.org/case-studies/thailands-universalcoverage-scheme.

47. Topothai T, Chandrasiri O, Liangruenrom N, Tangcharoensathien V. Renewing commitments to physical activity targets in Thailand. Lancet. 2016;388(10051):1258-60

48. Division of Physical Activity and Health. Thai national strategic plan on promotion of physical activity (2018-2030). Nonthaburi: Division of Physical Activity and Health, Ministry of Public Health; 2017.

49. 10th National Health Assembly. Promotion of physical activity for Thai people at all age groups; 2017. 7 March 2019. Available from: https://en. nationalhealth.or.th/wp-content/uploads/2017/11/NHA10-Miain-Documentpromotion-of-physical-activity_PA.pdf.

50. World Health Organization. Global recommendations on physical activity for health. Geneva: World Health Organization; 2010.

51. Liangruenrom N, Suttikasem K, Craike M, Bennie JA, Biddle SJH, Pedisic Z. Physical activity and sedentary behaviour research in Thailand: a systematic scoping review. BMC Public Health. 2018;18(1):733.

52. Hoyos C, Glozier N, Marshall NS. Recent evidence on worldwide trends on sleep duration. Curr Sleep Med Rep. 2015;1 (4):195-204.

53. Matricciani L, Bin YS, Lallukka T, Kronholm E, Dumuid D, Paquet C, et al. Past, present, and future: trends in sleep duration and implications for public health. Sleep Health. 2017;3(5):317-23.

54. Youngstedt SD, Goff EE, Reynolds AM, Kripke DF, Irwin MR, Bootzin RR, et al. Has adult sleep duration declined over the last 50+ years? Sleep Med Rev. 2016;28:69-85.

55. National Statistical Office. The time use survey 2015. Bangkok: National Statistical Office, Ministry of Information and Communication Technology; 2016. Report no.: ISBN 978-974-11-3056-6.

56. National Statistical Office. The time use survey 2009. Bangkok: National Statistical Office, Ministry of Information and Communication Technology; 2011. Report no.: ISBN 978-974-11-3056-6.

57. National Statistical Office. The time use survey 2001. Bangkok: National Statistical Office; 2002.

58. National Statistical Office. The time use survey 2004. Bangko: National Statistical Office, Ministry of Information and Communication Technology; 2005.

59. National Statistical Office. About the National Statistical Office. Bangkok: National Statistical Office; 2004. Available from: http://web.nso.go.th/en/abt. htm.

60. United Nations Statistics Division (UNSD). Guide to producing statistics on time use: measuring paid and unpaid work. New York: Department of Economic and Social Affairs, United Nations; 2005.

61. United Nations Statistics Division (UNSD). International classification of activities for time use statistics 2016 (ICATUS 2016). New York: Department of Economic and Social Affairs, United Nations; 2017.

62. Ministry of Labour. Labour Protection Act (No. 6), B.E. 2560 (2017). Bangkok: Ministry of Labour, Government of Thailand; 2017.

63. Olds T, Burton NW, Sprod J, Maher C, Ferrar K, Brown WJ, et al. One day you'll wake up and won't have to go to work: the impact of changes in time use on mental health following retirement. PLoS One. 2018;13(6): e0199605.

64. Liangruenrom N, Craike M, Dumuid D, Biddle S, Tudor-Locke C, Ainsworth BE, et al. Standardised criteria for classifying the International Classification of Activities for Time-Use Statistics (ICATUS) activity groups into sleep, sedentary behaviour, and physical activity. Int I Behav Nutr Phys Act. 2019;16:106.

65. Tudor-Locke C, Washington TL, Ainsworth BE, Troiano RP. Linking the American Time Use Survey (ATUS) and the compendium of physical activities: methods and rationale. J Phys Act Health. 2009:6(3):347-53.

66. Deyaert J, Harms T, Weenas D, Gershuny J, Glorieux I. Attaching metabolic expenditures to standard occupational classification systems: perspectives from time-use research. BMC Public Health. 2017;17(1):620.

67. Bauman A, Reis RS, Sallis JF, Wells JC, Loos RJ, Martin BW, et al. Correlates of physical activity: why are some people physically active and others not? Lancet. 2012;380(9838):258-71.

68. Sallis JF, Bull F, Guthold R, Heath GW, Inoue S, Kelly P, et al. Progress in physical activity over the Olympic quadrennium. Lancet. 2016;388(10051): 1325-36.

69. Trost SG, Owen N, Bauman AE, Sallis JF, Brown W. Correlates of adults' participation in physical activity: review and update. Med Sci Sports Exerc. 2002;34(12):1996-2001.
70. Koyanagi A, Stubbs B, Vancampfort D. Correlates of low physical activity across 46 low-and middle-income countries: a cross-sectional analysis of community-based data. Prev Med. 2018;106:107-13.

71. Koyanagi A, Stubbs B, Vancampfort D. Correlates of sedentary behavior in the general population: a cross-sectional study using nationally representative data from six low- and middle-income countries. PLoS One. 2018;13(8):e0202222

72. Bennie JA, Chau JY, van der Ploeg HP, Stamatakis E, Do A, Bauman A. The prevalence and correlates of sitting in European adults-a comparison of 32 Eurobarometer-participating countries. Int J Behav Nutr Phys Act. 2013;10(1): 107.

73. Kim KH, Sobal J. Religion, social support, fat intake and physical activity. Public Health Nutr. 2004;7(6):773-81.

74. Langøien LJ, Terragni L, Rugseth G, Nicolaou M, Holdsworth M, Stronks K, et al. Systematic mapping review of the factors influencing physical activity and sedentary behaviour in ethnic minority groups in Europe: a DEDIPAC study. Int J Behav Nutr Phys Act. 2017;14(1):99.

75. Viechtbauer W. Conducting meta-analyses in $\mathrm{R}$ with the metafor package. J Stat Softw. 2010;36(3):1-48.

76. Barnier J, Briatte F, Larmarange J. questionr: functions to make surveys processing easier. R package version 0.7. 0; 2018.

77. Guthold R, Stevens GA, Riley LM, Bull FC. Worldwide trends in insufficient physical activity from 2001 to 2016: a pooled analysis of 358 populationbased surveys with 1. 9 million participants. Lancet Glob Health. 2018;6(10): e1077-e86.

78. World Bank Group. Thailand Overview 2018; 2020. Available from: https:// www.worldbank.org/en/country/thailand/overview.

79. Baron KG, Reid K, Zee PC. Exercise to improve sleep in insomnia: exploration of the bidirectional effects. J Clin Sleep Med. 2013;9(8):819-24.

80. Dolezal BA, Neufeld EV, Boland DM, Martin JL, Cooper CB. Interrelationship between sleep and exercise: a systematic review. Adv Prev Med. 2017;2017: 1364387.

81. Murray K, Godbole S, Natarajan L, Full K, Hipp JA, Glanz K, et al. The relations between sleep, time of physical activity, and time outdoors among adult women. PLoS One. 2017:12(9):e0182013.

82. Tsunoda K, Kitano N, Kai Y, Uchida K, Kuchiki T, Okura T, et al. Prospective study of physical activity and sleep in middle-aged and older adults. Am J Prev Med. 2015;48(6):662-73.

83. Division of Physical Activity \& Health DoH, Ministry of Public Health. Situation of physical activity/exercise among Thai people. Nonthaburi: Ministry of Public Health; 2015. Available from: https://sites.google.com/site/ exercisemoph/sthankarn-kar-xxk-kalang-kay.

84. Pedisic Z, Shrestha N, Loprinzi PD, Mehata S, Mishra SR. Prevalence, patterns, and correlates of physical activity in Nepal: findings from a nationally representative study using the Global Physical Activity Questionnaire (GPAQ). BMC Public Health. 2019;19(1):864.

85. Liangruenrom N, Craike M, Biddle SJH, Suttikasem K, Pedisic Z. Correlates of physical activity and sedentary behaviour in the Thai population: a systematic review. BMC Public Health. 2019;19(1):414.

86. Lenhart A, Purcell K, Smith A, Zickuhr K. Social media \& mobile internet use among teens and young adults. Millennials. Pew internet \& American life project. 2010.

87. Valle CG, Tate DF, Mayer DK, Allicock M, Cai J, Campbell MK. Physical activity in young adults: a signal detection analysis of Health Information National Trends Survey (HINTS) 2007 data. J Health Commun. 2015;20(2):134-46.

88. O'donoghue G, Perchoux C, Mensah K, Lakerveld J, Van Der Ploeg H, Bernaards $C$, et al. A systematic review of correlates of sedentary behaviour in adults aged 18-65 years: a socio-ecological approach. BMC Public Health. 2016:16(1):163.

89. Aadahl M, Andreasen AH, Hammer-Helmich L, Buhelt L, Jørgensen T, Glümer C. Recent temporal trends in sleep duration, domain-specific sedentary behaviour and physical activity. A survey among 25-79-year-old Danish adults. Scand J Public Health. 2013;41(7):706-11.

90. Ford ES, Cunningham TJ, Croft JBJS. Trends in self-reported sleep duration among US adults from 1985 to 2012. Sleep. 2015;38(5):829-32.

91. Pedišić Ž, Bauman A. Accelerometer-based measures in physical activity surveillance: current practices and issues. Br J Sports Med. 2015;49(4):219-23.

\section{Publisher's Note}

Springer Nature remains neutral with regard to jurisdictional claims in published maps and institutional affiliations. 